\title{
DECISIVE CREATURES AND LARGE CONTINUUM
}

\author{
JAKOB KELLNER* AND SAHARON SHELAH ${ }^{\dagger}$
}

\begin{abstract}
Aвstract. For $f, g \in \omega^{\omega}$ let $c_{f, g}^{\forall}$ be the minimal number of uniform $g$-splitting trees needed to cover the uniform $f$-splitting tree, i.e. for every branch $v$ of the $f$-tree, one of the $g$-trees contains $v . \quad c_{f, g}^{\exists}$ is the dual notion: For every branch $v$, one of the $g$-trees guesses $v(m)$ infinitely often.

It is consistent that $c_{f_{\epsilon}, g_{\epsilon}}^{\exists}=c_{f_{\epsilon}, g_{\epsilon}}^{\forall}=\kappa_{\epsilon}$ for $\aleph_{1}$ many pairwise different cardinals $\kappa_{\epsilon}$ and suitable pairs $\left(f_{\epsilon}, g_{\epsilon}\right)$.

For the proof we use creatures with sufficient bigness and halving. We show that the lim-inf creature forcing satisfies fusion and pure decision. We introduce decisiveness and use it to construct a variant of the countable support iteration of such forcings, which still satisfies fusion and pure decision.
\end{abstract}

\section{INTRODUCTION}

In the paper Many simple cardinal invariants [3], Goldstern and the second author construct a partial order $P$ that forces pairwise different values to $\aleph_{1}$ many instances of the cardinal characteristic $c_{f, g}^{\forall}$, defined as follows:

Let $f, g \in \omega^{\omega}$ (usually we have $f(n)>g(n)$ for all $\left.n\right)$. An $(f, g)$-slalom is a sequence $S=(S(n))_{n \in \omega}$ such that $S(n) \subseteq f(n)$ and $|S(n)| \leq g(n)$. A family $\mathcal{S}$ of $(f, g)$-slaloms is a $(\forall, f, g)$-cover, if for all $r \in \prod_{n \in \omega} f(n)$ there is an $S \in \mathcal{S}$ such that $r(n) \in S(n)$ for all $n \in \omega$. $c_{f, g}^{\forall}$ is the minimal size of a $(\forall, f, g)$-cover.

We investigate the dual notion: A family $\mathcal{S}$ of $(f, g)$-slaloms is an $(\exists, f, g)$-cover, if for all $r \in \prod_{n \in \omega} f(n)$ there is an $S \in \mathcal{S}$ such that $r(n) \in S(n)$ for infinitely many $n \in \omega . c_{f, g}^{\exists}$ is the minimal size of an $(\exists, f, g)$-cover.

In [3], the following is shown:

Assume that $\mathrm{CH}$ holds, that $\left(f_{\epsilon}, g_{\epsilon}\right)_{\epsilon \in \omega_{1}}$ are sufficiently different, and that $\kappa_{\epsilon}^{\aleph_{0}}=\kappa_{\epsilon}$ for all $\epsilon \in \omega_{1}$. Then there is a cardinal preserving partial order $P$ which forces that $c_{f_{\epsilon}, g_{\epsilon}}^{\forall}=\kappa_{\epsilon}$ for all $\epsilon \in \omega_{1}$.

Similar results regarding $c^{\exists}$ as well as a perfect set of invariants were promised to appear in a paper called $448 \mathrm{a}$, which never materialized. A result for continuum many different invariants of the form $c_{f_{\epsilon}, g_{\epsilon}}^{\forall}$ can be found in [4].

In this paper, we prove a version for countably many invariants $c^{\exists}$ :

Theorem 1. Assume that $\mathrm{CH}$ holds, that $\left(f_{\epsilon}, g_{\epsilon}\right)_{\epsilon \in \omega}$ are sufficiently different, and that $\kappa_{\epsilon}^{\aleph_{0}}=\kappa_{\epsilon}$ for all $\epsilon \in \omega$. Then there is a cardinal preserving, $\omega^{\omega}$-bounding partial order $P$ which forces that $c_{f_{\epsilon}, g_{\epsilon}}^{\exists}=c_{f_{\epsilon}, g_{\epsilon}}^{\forall}=\kappa_{\epsilon}$ for all $\epsilon \in \omega$.

Date: November 8, 2018

2000 Mathematics Subject Classification. 03E17;03E40.

* supported by a European Union Marie Curie EIF Fellowship, contract MEIF-CT-2006-024483.

$\dagger$ supported by the United States-Israel Binational Science Foundation (Grant no. 2002323), and by the US National Science Foundation grant NSF-DMS 0600940, publication 872. 
(See Section 7 for a definition of sufficiently different.)

We can also get $\omega_{1}$ many different invariants, but we do not know in the ground model which invariants will be picked:

Theorem 2. Assume that $\mathrm{CH}$ holds, and that $\kappa_{\epsilon}^{\aleph_{0}}=\kappa_{\epsilon}$ for all $\epsilon \in \omega_{1}$. Then there are pairs $\left(f_{v}, g_{v}\right)_{v \in \omega_{1}}$ and there is a cardinal preserving, $\omega^{\omega}$-bounding partial order $R$ which forces: For each $\epsilon \in \omega_{1}$ there is a $v(\epsilon) \in \omega_{1}$ such that $c_{v_{v(\epsilon)}, g_{v(\epsilon)}}^{\exists}=c_{f_{v(\epsilon)}, g_{v(\epsilon)}}^{\forall}=\kappa_{\epsilon}$.

In any case, if the $\kappa_{\epsilon}$ are pairwise different, then in the forcing extension there are infinitely many different cardinals below the continuum, i.e. $2^{\aleph_{0}}>\boldsymbol{\aleph}_{\omega}$. Therefore we cannot use countable support iterations. We cannot use finite support iterations either (otherwise we add many Cohen reals, which makes $c^{\forall}$ too big). Instead, we use a variant of the countable support product of lim-inf creature forcings. We do not assume that the reader knows anything about creature forcing. However, we do assume that the reader knows the definition of proper forcing (see e.g. [2] or, for the brave, [6]), and the fact that such forcings preserve $\omega_{1}$. Alternatively, it is sufficient to know Baumgartner's Axiom A (cf. [1]): it is easy to see that the forcings in this paper all satisfy Axiom A, and Axiom A forcings (are proper and therefore) preserve $\omega_{1}$.

We write $q \leq p$ to say that $q$ is stronger than $p$. We try to stick to Goldstern's alphabetic convention, i.e. whenever two conditions are compatible, the symbol used for the stronger condition comes lexicographically later.

The theorems in this paper are due to the second author. The first author's contribution was to fill in some details, to ask the second author to fill in other details, and to write the paper.

We thank a referee for very carefully reading the paper and pointing out a mistake and numerous unclarities.

Annotated contents. In the first part, we investigate lim-inf creature forcings:

Section 2 p. 3. We define the (one-dimensional) lim-inf creature forcing $\mathbb{Q}_{\infty}^{*}$.

Section 3 , p. 5. We use bigness and halving to show that $\mathbb{Q}_{\infty}^{*}$ satisfies pure decision (and fusion). This implies that $\mathbb{Q}_{\infty}^{*}$ is proper and $\omega^{\omega}$-bounding. We also show rapid reading of certain names. The proofs in this section will be generalized in Section 5 .

Section 4 p. 11 We introduce decisiveness and use it to extend bigness to functions defined on finite products of creatures. This allows us to show pure decision for finite products of lim-inf creature forcings.

Section 5 p. 13. We define the forcing $P$, a variant of the countable support product of lim-inf creature forcings, in such a way that the proof of Section 3 still works with only few changes. We also get $\boldsymbol{\aleph}_{2}$-cc (assuming $\mathrm{CH}$ ).

Section 6 p. 20. We show how to construct decisive creatures with sufficient bigness and halving.

In the second part, we use the methods of Section 5 to prove Theorems 1 and 2 ;

Section 7 p. 22. We formulate the requirements for Theorem 1 and define $P$, a variant the forcing in Section 5 ,

Section 8, p. 23. We show that $P_{\epsilon}$, a complete subforcing of $P$, adds a $c_{f_{\epsilon}, g_{\epsilon}}^{\forall}$-cover in $V\left[G_{P}\right]$. This proves $c_{f_{\epsilon}, g_{\epsilon}}^{\forall} \leq \kappa_{\epsilon}$.

Section 9 p. 25. We show that in $V\left[G_{P}\right]$ there can be no $c_{f_{\epsilon}, g_{\epsilon}}^{\exists}$-cover smaller than $\kappa_{\epsilon}$ : Otherwise we can find a condition $q$ that rapidly reads (without using index $\beta$ ) a slalom $\underset{\sim}{S}$ and forces that the generic real ${\underset{\sim}{\beta}}_{\beta}$ at $\beta$ meets $\underset{\sim}{S}$ infinitely often. 
We strengthen $q$ such that the possible values for the generic alway: $]$ avoid the slalom $S$, a contradiction.

Section 10, p. 27 We construct $\omega_{1}$ many suitable pairs $\left(f_{\epsilon}, g_{\epsilon}\right)$ the partial order $R$, a modification of $P$, to show Theorem 2

\section{LIM-INF CREATURE FORCINGS}

Creature forcing in general is described in the monograph Norms on possibilities I: forcing with trees and creatures [5] by Rosłanowski and the second author. The forcing of the proof in [3] can be interpreted as creature forcing as well, more specifically as a limsup tree creating creature forcing. We will use lim-inf creatures instead. These forcings are generally more complicated than the lim-sup case, and [5] shows that they can collapse $\omega_{1}$. In this paper, we will require increasingly strong bigness and halving, which guarantees pure decision and therefore properness.

We now describe the setting we use. Creature forcings are defined by a parameter, the creating pair $(\mathbf{K}, \boldsymbol{\Sigma})$. We use the following objects:

- A function $\mathbf{H}: \omega \rightarrow \omega \backslash\{0\}$.

- A strictly increasing function $\mathbf{F}: \omega \rightarrow \omega$ such that $\mathbf{F}(0)=0$.

- For every $n \in \omega$ a finite set $\mathbf{K}(n)$.

- For each $\mathfrak{c} \in \mathbf{K}(n)$, a real number nor(c) $\geq 0$, and a nonempty subset $\operatorname{val}(\mathfrak{c})$ of $\prod_{\mathbf{F}(n) \leq i<\mathbf{F}(n+1)} \mathbf{H}(i)$.

- We additionally require that $|\operatorname{val}(\mathfrak{c})|=1$ implies nor $(\mathfrak{c})=0$.

A $c \in \mathbf{K}(n)$ is called $n$-creature. The intended meaning of the $n$-creature $c$ is the following: the set of possible values for the generic object $\eta \in \prod_{i \in \omega} \mathbf{H}(i)$ restricted to the interval $[\mathbf{F}(n), \mathbf{F}(n+1)-1]$ is the set $\operatorname{val}(\mathfrak{c})$. nor(c) can be thought of measuring the amount of "freedom" the creature $c$ leaves on its interval. If $c$ determines its part of the generic real (i.e. if $\operatorname{val}(\mathfrak{c})$ is a singleton) then nor(c) $=0$ (i.e. $c$ leaves no freedom). However, this intuition about nor(c) has to be used with caution: In particular, $\operatorname{val}(\mathfrak{D}) \subseteq \operatorname{val}(\mathfrak{c})$ does generally not imply nor(D) $\leq \operatorname{nor}(\mathfrak{C})$.

We set $\mathbf{K}:=\bigcup_{n \in \omega} \mathbf{K}(n)$.

In our application we will use $\mathbf{F}(n)=n$, i.e. an $n$-creature lives on the singleton $\{n\}$.

We also have a function $\mathbf{\Sigma}: \mathbf{K} \rightarrow \mathcal{P}(\mathbf{K})$ satisfying:

- If $\mathfrak{c} \in \mathbf{K}(n)$ and $\boldsymbol{D} \in \mathbf{\Sigma}(\mathfrak{c})$ then $\mathfrak{D} \in \mathbf{K}(n)$.

- $\boldsymbol{\Sigma}$ is reflexive, i.e. $c \in \boldsymbol{\Sigma}(\mathfrak{c})$.

- $\boldsymbol{\Sigma}$ is transitive, i.e. $\boldsymbol{D} \in \boldsymbol{\Sigma}(\mathfrak{c})$ and $\boldsymbol{D}^{\prime} \in \boldsymbol{\Sigma}(\boldsymbol{D})$ implies $\boldsymbol{D}^{\prime} \in \boldsymbol{\Sigma}(\mathfrak{c})$.

- If $\mathfrak{D} \in \boldsymbol{\Sigma}(\mathfrak{C})$ then $\operatorname{val}(\mathfrak{D}) \subseteq \operatorname{val}(\mathfrak{c})$ and $\operatorname{nor}(\mathfrak{D}) \leq \operatorname{nor}(\mathfrak{c})$.

The intended meaning is that $\boldsymbol{\Sigma}(\mathfrak{c})$ is the set of creatures that are stronger than $c$.

To simplify notation later on, we extend the definitions of nor, val and $\boldsymbol{\Sigma}$ to sequences $s, t \in \prod_{\mathbf{F}(n) \leq i<\mathbf{F}(n+1)} \mathbf{H}(n)$ : We set

$$
\operatorname{nor}(t):=0, \quad \operatorname{val}(t):=\{t\}, \quad t \in \boldsymbol{\Sigma}(\mathfrak{c}) \text { iff } t \in \operatorname{val}(\mathfrak{c}), \quad s \in \boldsymbol{\Sigma}(t) \text { iff } s=t .
$$

We now define the lim-inf forcing $\mathbb{Q}_{\infty}^{*}(\mathbf{K}, \boldsymbol{\Sigma})$ :

Definition 2.1. A condition $p \in \mathbb{Q}_{\infty}^{*}(\mathbf{K}, \boldsymbol{\Sigma})$ consists of a trunk $t \in \prod_{i<\mathbf{F}(n)} \mathbf{H}(i)$ for some $n$ and a sequence $\left(\mathfrak{c}_{i}\right)_{i \geq n}$ such that $\mathfrak{c}_{i} \in \mathbf{K}(i)$ and $\operatorname{nor}\left(\mathfrak{c}_{i}\right)>0$ for all $i \geq n$, and $\lim \left(\operatorname{nor}\left(\mathfrak{c}_{i}\right)\right)=\infty$.

\footnotetext{
${ }^{1}$ This is the reason we have to use lim-inf creature forcing instead of lim-sup: When we deal with $c^{\forall}$, we have to "run away" from $\underset{\sim}{S}$ infinitely often, and it is enough to assume that we have sufficient space to do so infinitely often. But here we need sufficient space at every height.
} 


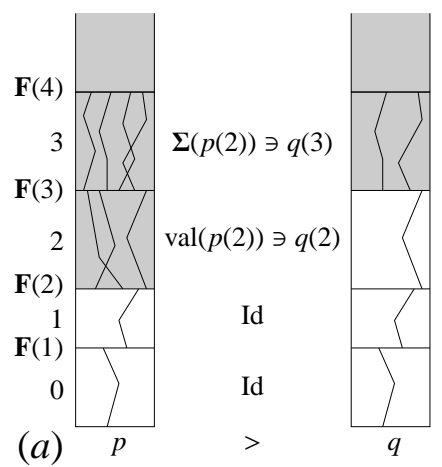

(b)

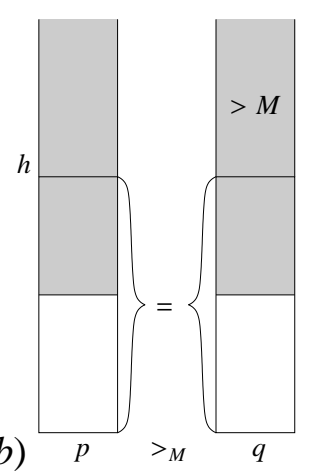

FIGURE 1. (a): $q \leq p, \operatorname{trnklh}(p)=2, \operatorname{trnklh}(q)=3$. (b): $q \leq_{M} p$

We set $\operatorname{trunk}(p):=t$, and the trunk-length $\operatorname{trnklh}(p):=n$, and we set

$$
p(i):= \begin{cases}\mathfrak{c}_{i} & \text { if } i \geq n, \\ t \uparrow[\mathbf{F}(i), \mathbf{F}(i+1)-1] & \text { otherwise. }\end{cases}
$$

So we can identify $p$ with the sequence $(p(i))_{i \in \omega}$. The order on $\mathbb{Q}_{\infty}^{*}$ is defined by $q \leq p$ if $\operatorname{trnklh}(q) \geq \operatorname{trnklh}(p)$ and $q(i) \in \boldsymbol{\Sigma}(p(i))$ for all $i$.

So in particular $q \leq p$ implies that $\operatorname{trunk}(q)$ extends $\operatorname{trunk}(p)$, see Figure 1 a).

Of course we assume that there are sufficiently large creatures, otherwise $\mathbb{Q}_{\infty}^{*}(\mathbf{K}, \boldsymbol{\Sigma})$ is empty

The forcing $\mathbb{Q}_{\infty}^{*}(\mathbf{K}, \boldsymbol{\Sigma})$ adds a generic real $\eta:=\bigcup_{p \in G} \operatorname{trunk}(p)$. Note that when we have halving (see next section), the generic filter $\tilde{G}$ is not determined by $\underset{\sim}{\eta}$, at least not in the usual way 3

A note on the requirement

$$
\operatorname{nor}(p(i))>0 \text { for each } i \geq \operatorname{trnklh}(p)
$$

in the definition of $\mathbb{Q}_{\infty}^{*}$ :

- We could drop 2.1], since in the resulting forcing notion the conditions that additionally satisfy (2.1) are dense anyway.

- Because of 2.11, we are really only interested in creatures with norm $>0$, so we could restrict ourselves to creating pairs containing only such creatures.

- Alternatively, we could omit the concept of trunk from the definition altogether. Instead, we could assume the following: For all $\mathfrak{c} \in \mathbf{K}(n)$ and all $s \in \operatorname{val}(\mathfrak{c})$ there is a $\mathfrak{D} \in \boldsymbol{\Sigma}(\mathfrak{C})$ such that $\operatorname{val}(\mathfrak{D})=\{s\}$ (and therefore nor $(\mathfrak{D})=0$ ). However, this is not the "right" way to think about creature forcing, and this version could not be generalized to our variant of the countable support product.

In the rest of the section, we briefly comment on how our setting fits into the framework of creature forcing developed in [5]:

\footnotetext{
${ }^{2}$ We need: For each $l \in \omega$ there is an $n \in \omega$ such that for all $m>n$ there is some $m$-creature with norm at least $l$.

${ }^{3}$ If $\operatorname{nor}(\mathfrak{c})$ is a function of $\operatorname{val}(\mathfrak{c})$ and $\operatorname{val}(\mathfrak{D}) \subseteq \operatorname{val}(\mathfrak{c})$ implies $\mathfrak{D} \in \boldsymbol{\Sigma}(\mathfrak{c})$, then the generic filter is determined by $\eta \underset{\sim}{\eta}$. This assumption is reasonable (and is satisfied in many creature forcing constructions), but it is incompatible with halving.
} 
A pair $(\mathbf{K}, \boldsymbol{\Sigma})$ as above is a creating pair as defined in [5, 1.2]. It satisfies the following additional properties:

- finitary [5, 1.1.3]: $\mathbf{H}(n)$ and $\boldsymbol{\Sigma}(c)$ are always finite.

- simple [5, 2.1.7]: $\boldsymbol{\Sigma}$ is defined on single creatures only 4

- forgetful [5, 1.2.5]: val(c) does not depend on values of the generic real outside of the interval of $\mathrm{c} 5$

- nice and smooth [5, 1.2.5]: A technical requirement that is trivial in the case of forgetful simple creating pairs.

In [5] two main frameworks for forcings are examined: creature forcings [5, 1.2.6] (defined by a creating pair [5, 1.2.2]) and tree creature forcings [5, 1.3.5] (defined via a tree-creating pair [5, 1.3.3]). So in this paper we deal with creature forcings 9

In [5] several ways to define forcings from a creating pair are introduced. One example is lim-sup creature forcing $\mathbb{Q}_{w \infty}^{*}$ defined in [5, 1.2.6]. Many simple cardinal invariants [3] uses (a countable support product of) such forcings. The lim-inf case $\mathbb{Q}_{\infty}^{*}$ is generally harder to handle, and [5, 1.4.5] proves that $\mathbb{Q}_{\infty}^{*}$ can collapse $\omega_{1}$. In the rest of [5], $\mathbb{Q}_{\infty}^{*}$ is only considered in a special case (incompatible with simple) where $\mathbb{Q}_{\infty}^{*}$ is actually equivalent to other forcings that are better behaved (cf. [5] p23 and 2.1.3]). We will introduce additional assumptions (increasingly strong bigness and halving) to guarantee that $\mathbb{Q}_{\infty}^{*}$ is proper and $\omega^{\omega}$-bounding. These assumptions will actually make $\mathbb{Q}_{\infty}^{*}$ similar to $\mathbb{Q}_{f}^{*}$ of [5].

\section{BIGNESS AND HALVING, PROPERNESS OF $\mathbb{Q}_{\infty}^{*}$}

We will now introduce properties that guarantee that $\mathbb{Q}_{\infty}^{*}$ is proper.

Definition 3.1. Let $0<r \leq 1, B \in \omega$.

- $\mathfrak{c}$ is $(B, r)$-big if for all functions $F: \operatorname{val}(\mathfrak{c}) \rightarrow B$ there is a $\mathfrak{D} \in \boldsymbol{\Sigma}(\mathfrak{c})$ such that $\operatorname{nor}(\triangleright) \geq \operatorname{nor}(\mathfrak{c})-r$ and $F\lceil\operatorname{val}(\mathfrak{D})$ is constant $\square$

- $\mathbf{K}(n)$ is $(B, r)$-big if every $\mathfrak{c} \in \mathbf{K}(n)$ with $\operatorname{nor}(\mathfrak{c})>1$ is $(B, r)$-big.

- $c$ is $r$-halving 8 if there is a half(c) $\in \boldsymbol{\Sigma}(\mathfrak{c})$ such that

- $\operatorname{nor}($ half $(\mathfrak{c})) \geq \operatorname{nor}(\mathfrak{c})-r$, and

- if $\mathfrak{D} \in \boldsymbol{\Sigma}($ half $(\mathfrak{c}))$ and nor( $(\mathfrak{D})>0$, then there is a $\mathfrak{D}^{\prime} \in \Sigma(\mathfrak{c})$ such that $\operatorname{nor}\left(\triangleright^{\prime}\right) \geq \operatorname{nor}(\mathfrak{C})-r$ and $\operatorname{val}\left(\triangleright^{\prime}\right) \subseteq \operatorname{val}(\mathfrak{D})$.

- $\mathbf{K}(n)$ is $r$-halving, if all $\mathfrak{c} \in \mathbf{K}(n)$ with nor(c) $>1$ are $r$-halving.

So given $c$ and $\boldsymbol{D} \in \boldsymbol{\Sigma}($ half $(\mathfrak{c}))$ as in the definition of halving, we can "un-halve" $\boldsymbol{D}$ to get $\mathfrak{D}^{\prime}$. Note that this $\mathfrak{D}^{\prime}$ generally is not in $\boldsymbol{\Sigma}($ half $(\mathfrak{C}))$, although $\operatorname{val}\left(\mathfrak{D}^{\prime}\right) \subseteq \operatorname{val}(\mathfrak{D}) \subseteq \operatorname{val}($ half $(\mathfrak{C})$ ).

\footnotetext{
${ }^{4}$ In non-simple creating pairs we can have something like $\boldsymbol{D} \in \boldsymbol{\Sigma}\left(\left\{\mathfrak{c}_{1}, \mathfrak{c}_{2}\right\}\right)$, e.g. $\mathfrak{c}_{1}$ could live on the interval $I_{1}$, $c_{2}$ on $I_{2}$, and $\triangleright d$ is $c_{1}$ and $c_{2}$ "glued together".

${ }^{5}$ In the general case, $\operatorname{val}(\mathfrak{c})$ is defined as a set of pairs $(u, v)$ where $v \in \prod_{i<\mathbf{F}(n+1)} \mathbf{H}(i)$ and $u=v \nmid \mathbf{F}(n)$. The intended meaning is that $c$ implies: If the generic object $\eta$ restricted to $\mathbf{F}(n)$ is $u$, then the possible values $v$ for $\eta \sim \mathbf{F}(n+1)$ are those $v$ such that $(u, v) \in \operatorname{val}(\mathfrak{c})$. Then "c is forgetful" is defined as: If $(u, v) \in \operatorname{val}(\mathfrak{c})$ and $u^{\prime} \in \prod_{i<\mathbf{F}(n)} \mathbf{H}(i)$ then $\left(u^{\prime}, v\right) \in \operatorname{val}(c)$. So in the forgetful case $\operatorname{val}(c)$ and $\{v:(\exists u)(u, v) \in \operatorname{val}(c)\}$ carry the same information. In this paper we call the latter set val(c), for simplicity of notation.

${ }^{6}$ Actually every simple forgetful creating pair can be interpreted as tree-creating pair as well. The resulting tree-forcing however is different from the creature forcing: the creature forcing corresponds to the "homogeneous" trees only.

${ }^{7}$ This is a variant of, but technically not quite the same as, [5] 2.2.1].

${ }^{8}$ cf. [5. 2.2.7]. The original definition used nor $($ half $(\mathfrak{c})) \geq \operatorname{nor}(\mathrm{c}) / 2$ instead of nor(c) $-r$, therefore the name halving.
} 
Every creature is $(1, r)$-big. If $r^{\prime}$ is smaller than $r$, then $\left(B, r^{\prime}\right)$-bigness implies $(B, r)$ bigness, and $r^{\prime}$-halving implies $r$-halving. We also get:

$$
\text { If } \mathfrak{c} \text { is }(B, r) \text {-big and } 0<r<\operatorname{nor}(\mathfrak{c}) \text {, then } B<|\operatorname{val}(\mathfrak{c})| \text {. }
$$

An example for creatures with bigness and halving (and the much stronger property decisiveness) can be found in Section 6

We now show that increasing bigness and halving implies properness:

Theorem 3.2. Set $\varphi(<n):=\prod_{i<\mathbf{F}(n)} \mathbf{H}(i)$ and $r(n):=1 /(n \varphi(<n))$. If $\mathbf{K}(n)$ is $(2, r(n))$-big and $r(n)$-halving for all $n$, then $\mathbb{Q}_{\infty}^{*}(\mathbf{K}, \mathbf{\Sigma})$ is $\omega^{\omega}$-bounding and proper and preserves the size of the continuum (in the following sense: in the extension, there is a bijection between the reals and old reals).

So in particular, $\mathrm{CH}$ is preserved.

Note 3.3. Only the growth rate of $r$ is relevant here. In particular: Fix some $\delta>1$. Then the theorem remains valid if we replace $(2, r(n))$-big and $r(n)$-halving with the weaker condition $(2, \delta \cdot r(n))$-big and $\delta \cdot r(n)$-halving. Also, it does not make any difference if we require bigness and halving only for those creatures with norm bigger than $\delta$ (instead of for all creatures with norm bigger than 1).

Note that $\varphi(<n)$ is the number of possible values for $\underset{\sim}{\uparrow} \mathbf{F}(n)$, or equivalently the number of possible trunks with trunk-length $n$.

We also set $\varphi(\leq n)=\varphi(<n+1)$ and $\varphi(=n)=\varphi(\leq n) / \varphi(<n)=\prod_{\mathbf{F}(n) \leq i<\mathbf{F}(n+1)} \mathbf{H}(i)$.

In the rest of this section we set $P=\mathbb{Q}_{\infty}^{*}(\mathbf{K}, \boldsymbol{\Sigma})$.

We use a standard pure decision argument:

Let $\operatorname{val}(p,<n)$ denote $\prod_{i<n} \operatorname{val}(p(i)$ ), the set of possible values (modulo $p$ ) for $\underset{\sim}{\eta} \uparrow \mathbf{F}(n)$. The size of this set is at most $\varphi(<n)$.

We define for every $s \in \prod_{i<\mathbf{F}(n)} \mathbf{H}(i)$ a condition $p \wedge s: \operatorname{trnklh}(p \wedge s)=\max (n, \operatorname{trnklh}(p))$, and

$$
(p \wedge s)(i):= \begin{cases}s \uparrow[F(i), F(i+1)-1] & \text { if } i<n \\ p(i) & \text { otherwise. }\end{cases}
$$

We use this notion mostly for $s \in \operatorname{val}(p,<n)$. In this case, $p \wedge s \leq p$. Note that

$$
\{p \wedge s: s \in \operatorname{val}(p,<n)\} \text { is predense under } p,
$$

which implies for all $s \in \operatorname{val}(p,<n)$

$$
p \wedge s \Vdash \varphi \text { iff } p \Vdash(s<\underset{\sim}{\eta} \rightarrow \varphi) .
$$

$q \leq^{*} p$ means that $q$ forces $p$ to be in the generic filter.

$$
q \leq^{*} p \text { implies } \operatorname{val}(q,<n) \subseteq \operatorname{val}(p,<n) .
$$

It is important to note that $\operatorname{val}(q(i)) \subseteq \operatorname{val}(p(i))$ for all $i$ does not imply $q \leq^{*} p$ (or even just $q \| p$ ), since $\operatorname{val}(\mathfrak{D}) \subseteq \operatorname{val}(\mathfrak{c})$ does not imply $\mathfrak{b} \in \boldsymbol{\Sigma}(\mathfrak{c})$. (This would contradict halving.) However, the following does follow from (3.2):

(3.5) If $\operatorname{val}(q(i)) \subseteq \operatorname{val}(p(i))$ for all $i \leq h$ and $q(i) \in \Sigma(p(i))$ for all $i>h$, then $q \leq^{*} p$.

Let $\tau$ be a name of an ordinal. $p<n$-decides $\tau$, if $p \wedge s$ decides $\int \tau$ for all $s \in \operatorname{val}(p,<n)$. $q$ essentially decides $\underset{\sim}{\tau}$, if $p<n$-decides $\underset{\sim}{\tau}$ for some $n$.

\footnotetext{
9i.e. there is an $\alpha_{s} \in V$ such that $p \wedge s$ forces $\underset{\sim}{\tau}=\check{\alpha}_{s}$.
} 
So if $p$ essentially decides $\underset{\sim}{\tau}$, then we can calculate the value of $\underset{\sim}{\tau}$ from a finite set of possible trunks of $p$. So (3.3) and (3.4) imply:

$$
\text { If } p<n \text {-decides } \underset{\sim}{\tau} \text {, and } q \leq^{*} p \text {, then } q<n \text {-decides } \underset{\sim}{\tau} \text {. }
$$

We also get:

(3.7) If $q \wedge s$ essentially decides $\tau$ for each $s \in \operatorname{val}(q,<n)$, then so does $q$.

We define the following (non-transitive) relations $\leq_{n}(n \in \omega)$ on $P$ :

$$
\begin{aligned}
& q \leq_{n} p \text { if } q \leq p \text { and there is an } h \geq n \text { such that } q \uparrow h=p \uparrow h \text { and } \\
& \operatorname{nor}(q(i)) \geq n \text { for all } i \geq h .
\end{aligned}
$$

(Cf. Figure1 b) on page 4).

Proof of Theorem 3.2 We will show the following properties:

- $q \leq_{0} p$ implies $q \leq p$, and $q \leq_{n+1} p$ implies $q \leq_{n} p$.

- (Fusion.) For every sequence $p_{0} \geq_{0} p_{1} \geq_{1} p_{2} \geq \ldots$ there is a $q$ stronger than each $p_{n}$.

- (Pure decision.) For every name $\tau$ of an ordinal, $n \in \omega$, and $p \in P$, there is a $q \leq_{n} p$ essentially deciding $\tau$.

Then the standard argument can be employed to show Theorem 3.2

- $\omega^{\omega}$-bounding: Let $f$ be the name for a function from $\omega$ into ordinals and $p \in P$. Set $p_{0}=p$. If $p_{n}$ is already constructed, choose $p_{n+1} \leq_{n+1} p_{n}$ essentially deciding $f(n)$. Fuse the sequence into some $q$. Then modulo $q$ there are only finitely many possibilities for each $f(n)$.

- Proper: Let $N<H \tilde{(} \chi)$ be countable and contain $P$ and $p_{0}$. Let $\left(\tau_{n}\right)_{n \in \omega}$ list the $P$-names of ordinals that are in $N$. Choose (in $N$ ) $p_{n+1} \leq_{n} p_{n}$ such that $p_{n+1}$ essentially decides $\tau_{n}$. If $q \leq p_{n}$ for all $n$, then $q$ is is $N$-generic.

- The size of the continuum: So for every $p$ in $P$ and $P$-name $r$ for a real there is a $q \leq p$ continuously reading $\underset{\sim}{r}$. This means that $\underset{\sim}{r}$ is calculated by a function

$$
\text { eval : } \bigcup_{n \in \omega} \operatorname{val}(q,<n) \rightarrow 2^{<\omega} \text {. }
$$

(Since each $\underset{\sim}{r}(m)$ is determined by $\operatorname{val}(q,<M)$ for some $M$.) There are only $2^{\aleph_{0}}$ many such functions, and $|P|=2^{\aleph_{0}}$ many conditions.

So we just have to show pure decision and fusion. Fusion is easy: Let $\left(p_{n}\right)_{n \in \omega}$ satisfy $p_{n+1} \leq_{n+1} p_{n}$. Set $q(n)=p_{n}(n)$. Then $q$ is in $P$ : Fix any $M \in \omega$. There is an $h>M$ such that

$$
\operatorname{nor}\left(p_{M}(m)\right) \geq M \text { for all } m \geq h \text {. }
$$

Then (3.9) holds for $p_{M+1}$ as well, and for each $p_{k}$ with $k>M$, and therefore for $q$. Clearly, $q \leq p_{n}$ for each $n$.

It remains to be shown that $P$ satisfies pure decision.

Let $\tau$ be the name of an ordinal.

The basic construction $S(p, M)$ :

Assume that $\operatorname{trnklh}(p)=n$ and $M \in \omega$. We define $S(p, M)$ the following way, see Figure2

Enumerate $\operatorname{val}(p, \leq n)$ as $s^{0}, \ldots, s^{l-1}$. So $l \leq \varphi(=n)$. Set $p^{-1}=p$. Given $p^{k}$, define $p^{k+1} \in P$ as follows: $\operatorname{trunk}\left(p^{k+1}\right)=s^{k+1}, p^{k+1} \leq p^{k} \wedge s^{k+1}$, and there is an $h^{k+1}$ such that

- if $n<m<h^{k+1}$, then $\operatorname{nor}\left(p^{k+1}(m)\right) \geq \operatorname{nor}\left(p^{k}(m)\right)-r(m)$,

- if $m \geq h^{k+1}$, then $\operatorname{nor}\left(p^{k+1}(m)\right) \geq M$,

and such that additionally one of the following two cases holds: 


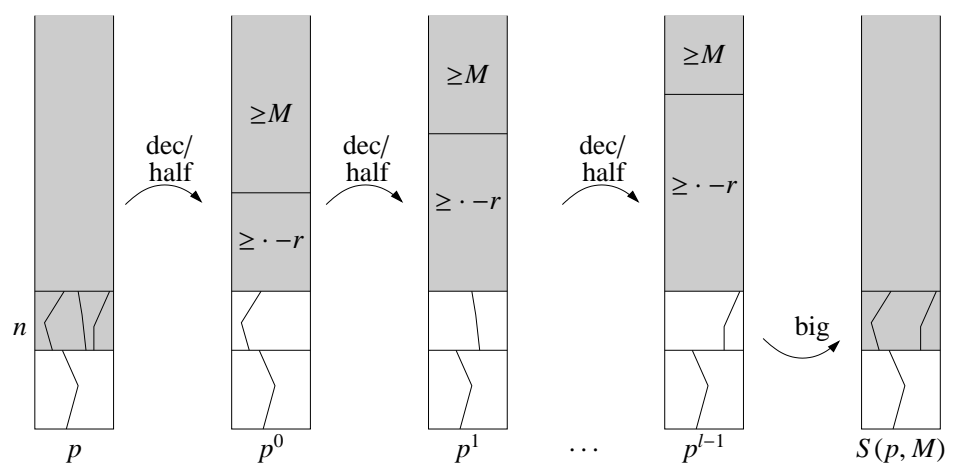

Figure 2. The basic construction $S(p, M)$.

dec: $p^{k+1}$ essentially decides $\underset{\sim}{\tau}$, or

half: it is not possible to satisfy "dec" (for any choice of $h^{k+1}$ ), then $p^{k+1}(m)=$ half $\left(p^{k}(m)\right)$ for all $m>n$.

This way we construct $p^{k}$ for each $0 \leq k<l$. At each step $0 \leq k<l$, we have one of the cases "dec" or "half". This gives a function $F: \operatorname{val}(p(n)) \rightarrow\{$ dec, half $\}$, and we use bigness to thin out $p(n)$ and get some $\mathfrak{D} \in \boldsymbol{\Sigma}(p(n))$ such that $F \uparrow \operatorname{val}(\mathfrak{D})$ is constant and

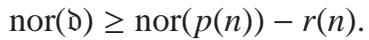

Note that in this construction we have to assume that $\operatorname{nor}\left(p^{k}(m)\right)>1$ for all $-1 \leq k<$ $l-1$ and $m>n$, otherwise we cannot halve $p^{k}(m)$. Also, nor $(p(n))$ has to be bigger than 1 , otherwise we cannot use bigness. Let $S(p, M)$ be undefined if these conditions are not met. Otherwise, we define $q=S(p, M)$ as follows:

$$
q \uparrow n=p \uparrow n=\operatorname{trunk}(p), \quad q(n)=\mathrm{D}, \quad q(m)=p^{l-1}(m) \text { for } m>n .
$$

We call $q$ halving, if the constant value of $F \uparrow \operatorname{val}(q(n))$ is "half". We will show that $q$ cannot be halving.

If $q$ is not halving, i.e. if the constant value is "dec", then $q$ essentially decides $\underset{\sim}{\tau}$ : If $s \in \operatorname{val}(q, \leq n)$, then $s=s^{k}$ for some $k<l$, and $q \wedge s \leq p^{k}$ essentially decides $\underset{\sim}{\tau}$. Now use (3.7).

Some properties of $S(p, M)$ :

If $q=S(p, M)$ is defined, then it satisfies the following:

$$
\begin{gathered}
\operatorname{nor}(q(n)) \geq \operatorname{nor}(p(n))-r(n) . \\
\text { If } m>n \text {, then } \operatorname{nor}(q(m)) \geq \min (M, \operatorname{nor}(p(m)))-\varphi(=n) \cdot r(m) . \\
\text { If } q \text { is halving, then no } q^{\prime} \leq q \text { with trunk-length } n+1 \text { essentially decides } \underset{\sim}{\tau} \text {. }
\end{gathered}
$$

To see (3.12), assume that $q^{\prime}$ is a counterexample. So $q^{\prime} \leq q \wedge s^{k} \leq p^{k}$ for some $0 \leq k<l$, and $\operatorname{nor}\left(q^{\prime}(m)\right)>0$ for all $m>n$. Since $q$ is halving, $p^{k}$ was produced by halving $p^{k-1}$. Pick an $h$ such that $\operatorname{nor}\left(q^{\prime}(m)\right)>M$ for all $m \geq h$. For $n<m<h, p^{k}(m)=\operatorname{half}\left(p^{k-1}(m)\right)$ and $q^{\prime}(m) \in \boldsymbol{\Sigma}(q(m)) \subseteq \boldsymbol{\Sigma}\left(p^{k}(m)\right)$, so we can un-halve $q^{\prime}(m)$ to get some $\mathfrak{D}_{m} \in \boldsymbol{\Sigma}\left(p^{k-1}(m)\right)$ with $\operatorname{val}\left(\mathfrak{D}_{m}\right) \subseteq \operatorname{val}\left(q^{\prime}(m)\right)$ and $\operatorname{nor}\left(\mathfrak{D}_{m}\right) \geq \operatorname{nor}\left(p^{k-1}(m)\right)-r(m)$. But then we could have chosen a deciding condition $r$ instead of $p^{k}$ : Define $r(m)=\delta_{m}$ for $n<m<h$ and $r(m)=$ $q^{\prime}(m)$ otherwise. According to (3.5), $r \leq^{*} q$. 3.6 implies that $r$ essentially decides $\underset{\sim}{\tau}$, a contradiction. 
$S(p, M)$ essentially decides:

We assume that $S(p, M)$ is halving and get a contradiction the following way: We show that the "successors" of $q$ with increased stem have to be halving as well, and we can fuse them into some $q^{\omega}$. But there will be a $q^{\prime} \leq q^{\omega}$ deciding $\underset{\sim}{\tau}$, a contradiction. In more detail:

If $\operatorname{trnklh}(p)=n, \operatorname{nor}(p(m))>3$ for all $m \geq n$ and if $M>3$, then $S(p, M)$ exists and is not halving.

Assume towards a contradiction that $S(p, M)$ is halving (or does not exist). Set $q^{n-1}=p$. Assume that for $k \geq n-1$, we have already defined $q^{k}$. We set $M_{k}=M+k+1-n$ (note that $\left.M_{n-1}=M\right)$, and define $q^{k+1}$ the following way:

List $\operatorname{val}\left(q^{k}, \leq k\right)$ as $s^{0}, \ldots, s^{l-1}$. So $l \leq \varphi(\leq k)$. Set $r^{-1}=q^{k}$. Given $r^{i-1}$, set

$$
r^{i}=S\left(r^{i-1} \wedge s^{i}, M_{k}\right)
$$

(if defined). So $r^{i}$ has trunk-length $k+1$. Define $q^{k+1}(m)$ to be $q^{k}(m)$ for $m \leq k$ and $r^{l-1}(m)$ otherwise.

So in particular, $q^{n}=S(p, M)$.

If $q^{k+1}$ is defined, then 3.10) and (3.11) imply:

- $q^{k+1}(m)=q^{k}(m)$ for $m \leq k$.

- $\operatorname{nor}\left(q^{k+1}(k+1)\right) \geq \operatorname{nor}\left(q^{k}(k)\right)-\varphi(\leq k) \cdot r(k+1)$.

- $\operatorname{nor}\left(q^{k+1}(m)\right) \geq \min \left(M_{k}, \operatorname{nor}\left(q^{k}(m)\right)\right)-\varphi(\leq k+1) \cdot r(m)$ for $m>k+1$.

So in any case, we get for all $m \in \omega$

$$
\operatorname{nor}\left(q^{k+1}(m)\right) \geq \min \left(M_{k}, \operatorname{nor}\left(q^{k}(m)\right)\right)-\varphi(<m) \cdot r(m) .
$$

Iterating this $l$ many steps (note that $q^{k}(m)$ remains constant if $k \geq m$ ) we get for all $m$ :

$$
\operatorname{nor}\left(q^{k+l}(m)\right) \geq \min \left(M_{k}, \operatorname{nor}\left(q^{k}(m)\right)\right)-\min (l, m-k) \cdot \varphi(<m) \cdot r(m),
$$

and since $r(m)=1 /(m \cdot \varphi(<m))$, we get

$$
\operatorname{nor}\left(q^{k+l}(m)\right) \geq \min \left(M_{k}, \operatorname{nor}\left(q^{k}(m)\right)\right)-1 \text {. }
$$

If we set $k=n-1$, this shows that $\operatorname{nor}\left(q^{k+l}(m)\right) \geq 2$ for all $l \in \omega$, and that therefore $q^{k+l+1}$ is defined. Also, if we define $q^{\omega}$ by $q^{\omega}(m)=q^{m}(m)$, then $q^{\omega} \in P$ : Given $N \in \omega$, pick $k$ such that $M_{k}>N+1$ and pick $h>k$ such that $\operatorname{nor}\left(q^{k}(m)\right)>N+1$ for all $m>h$. If $m>h$, i.e. $m=$ $k+l$ for some $l>0$, then $q^{\omega}(m)=q^{k+l}(m)$, and $\operatorname{nor}\left(q^{k+l}(m)\right) \geq \min \left(M_{k}, \operatorname{nor}\left(q^{k}(m)\right)-1>N\right.$.

Also, $q^{\omega} \leq q^{k}$ for all $k \in \omega$.

The property 3.12 of $S$ can by induction be generalized to any $k \geq n$ (recall that $\left.q=S(p, M)=q^{n}\right)$.

No $q^{\prime} \leq q^{k}$ with trunk-length $k+1$ essentially decides $\underset{\sim}{\tau}$.

For $k=n$ this is 3.12). We assume that 3.18 holds for $k$ and show it for $k+1$. Assume $q^{\prime}$ is a counterexample. $q^{\prime}$ is stronger than some of the $r^{i}(0 \leq i<l)$ used in (3.14) to construct $q^{k+1} . r^{i}=S\left(r^{i-1} \wedge s^{i}, M_{k}\right)$ has trunk-length $k+1$ and is stronger than $q^{k}$, so we can apply (3.18) to see that $r^{i}$ cannot essentially decide $\underset{\sim}{\tau}$. So $r^{i}$ is halving. Using (3.12), we see that no $q^{\prime} \leq r^{i}$ with trunk-length $k+2$ essentially decides $\underset{\sim}{\tau}$, a contradiction.

On the other hand, there is a $q^{\prime} \leq q^{\omega}$ deciding $\underset{\sim}{\tau}$. Set $k=\operatorname{trnklh}(q)-1$. Then $q^{\prime} \leq q^{\omega} \leq q^{k}$ contradicts 3.18.

\section{Pure decision:}

Given $p \in P$ and $M \in \omega$, pick $n$ such that $p(m)>M+5$ for all $m \geq n$. Similarly to above, enumerate $\operatorname{val}(p,<n)$ as $s^{0}, \ldots, s^{l-1}$, set $r^{-1}=p$ and $r^{k+1}=S\left(r^{k} \wedge s^{k+1}, M+5\right)$. Define $q$ by $q \uparrow n=p \uparrow n$ and $q(m)=r^{l-1}(m)$ for $m \geq n$. Just as in 3.15, $\operatorname{nor}(q(m)) \geq$ 
$\min (M+5, \operatorname{nor}(p(m)))-1>M+4$ for $m>n$, i.e. $q \leq_{M} p$. As we already know by 3.13, each $r^{k}$ essentially decides $\mathcal{\sim}$, so by [3.7), $q$ essentially decides $\tau$ as well.

A simple modification of the proof leads to a stronger property: Using the same $\varphi$ and $r$ as in the previous theorem, we get:

Theorem 3.4. Assume that $g: \omega \rightarrow \omega \backslash 1$ is monotonously increasing, that $\underset{v}{\underline{x}}$ is a $P$-name and that $p \in P$ forces that $\underset{\sim}{\gamma(n)}<g(n)$ for all $n$. If each $\mathbf{K}(n)$ is $(g(n)+1, r(n))$-big and $r(n)$-halving, then there is a $q \leq p$ which $<n$-decides $\underset{y}{(n)}$ for all $n$.

We call this phenomenon rapid reading.

Proof. We modify the last proof in the following way:

The basic construction $S(p, l, M)$ : We again assume that $n=\operatorname{trnklh}(p)$, and use the notation $S(p, l, M)$ (for $l \leq n$ ) for the same construction as $S(p, M)$, where we set $\tau=\underset{\sim}{q}(l)$, and instead of trying to essentially decide $\tau$, we try to decide it. So instead of the two cases "dec" and "half", we get $g(l)+1$ many cases: " $0 ", \ldots$, " $g(l)-1$ ", and (if none of these cases can be satisfied) "half". Since $l \leq n$ and $g$ is increasing, we can use $(g(n)+1, r(n))$-bigness instead of just $(2, r(n))$-bigness, and we again get a homogeneous D. If $S(p, l, M)$ is not halving, then it decides $y(l)$.

Some properties of $S(p, l, M)$ : We again get (3.10) and (3.11), and in (3.12) we replace "essentially decides $\underset{\sim}{ }$ ” with "decides $\underset{\sim}{\gamma(l)}$ ", i.e. we get:

If $q$ is halving, then no $q^{\prime} \leq q$ with trunk-length $n+1$ decides $\underset{\sim}{v}(l)$.

$S(p, l, M)$ decides: We again construct $q^{k}$, each time trying to decide $\tau=g(l)$ (independently of $k$ ). So (3.14) now reads:

$$
r^{i}=S\left(r^{i-1} \wedge s^{i}, l, M_{k}\right) .
$$

(Here we only need $(g(l)+1, r(k))$-bigness). Again we get (3.17), and therefore each $q^{k}$ (and $q^{\omega}$ ) is defined, and $(3.18)$ now tells us

$$
\text { No } q^{\prime} \leq q^{k} \text { with trunk-length } k+1 \text { decides } \underset{\sim}{ } \text {. }
$$

But there is some $q^{\prime} \leq q^{\omega}$ deciding $\tau$, a contradiction.

So far we know the following:

$$
\begin{aligned}
& \text { If } \operatorname{trnklh}(p)=n, \operatorname{nor}(p(m))>3 \text { for } m \geq n, \text { and } M>3 \text {, then } S(p, n, M) \\
& \text { exists and decides } \underset{\sim}{y}(n) .
\end{aligned}
$$

Rapid reading: Instead of the part on pure decision, we proceed as follows: Given $p \in P$, we can assume (by enlarging the stem) that $\operatorname{nor}(p(m))>5$ for all $m>\operatorname{trnklh}(p)$. We set $k_{0}=\operatorname{trnklh}(p)-1$ and $q^{k_{0}}=p^{\prime}$. We now construct $q^{k}$ and $q^{\omega}$ just as above, but this time using

$$
r^{i}=S\left(r^{i-1} \wedge s^{i}, k+1, M_{k}\right) .
$$

As in 3.17) we see that $r^{i}, q^{k}$ and $q^{\omega}$ exist. $r^{i}$ has sufficient norm and trunk-length $k+1$,

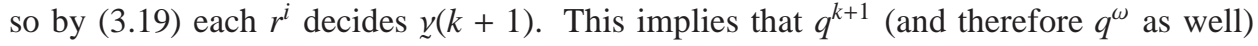
$\leq k$-decides $\underset{\sim}{\gamma}(k+1)$.

Note that $P$ has size continuum, and in particular it is $\left(2^{\aleph_{0}}\right)^{+}$-cc. Together with proper, that gives us:

Lemma 3.5. Under $\mathrm{CH}$ and the assumptions of Theorem 3.2 P preserves all cardinals (and cofinalities) and the size of the continuum. 
4. DECISIVENESS, PROPERNESS OF FINITE PRODUCTS

In this section, we fix a finite set $I$ and for every $i \in I$ a creating pair $\left(\mathbf{K}_{i}, \boldsymbol{\Sigma}_{i}\right)$.

The product forcing $\prod_{i \in I} \mathbb{Q}_{\infty}^{*}\left(\mathbf{K}_{i}, \boldsymbol{\Sigma}_{i}\right)$ is equivalent to $\mathbb{Q}_{\infty}^{*}\left(\mathbf{K}_{I}, \boldsymbol{\Sigma}_{I}\right)$, where the creating pair $\left(\mathbf{K}_{I}, \boldsymbol{\Sigma}_{I}\right)$ is defined as follows: An $n$-creature $\mathfrak{c} \in \mathbf{K}_{I}(n)$ corresponds to an $|I|$-tuple $\left(\mathfrak{c}_{i}\right)_{i \in I}$ such that $\mathfrak{c}_{i} \in \mathbf{K}_{i}(n) . \operatorname{val}(\mathfrak{c})=\prod_{i \in I} \operatorname{val}\left(\mathfrak{c}_{i}\right), \operatorname{nor}(\mathfrak{c})=\min \left(\left\{\operatorname{nor}\left(\mathfrak{c}_{i}\right): i \in I\right\}\right)$, and $\mathfrak{D}=\left(\mathfrak{b}_{i}\right)_{i \in I}$ is in $\boldsymbol{\Sigma}(\mathfrak{c})$ if $\mathfrak{D}_{i} \in \boldsymbol{\Sigma}\left(\mathfrak{c}_{i}\right)$ for all $i \in I 10$

If each $\mathbf{K}_{i}(n)$ is $r$-halving, then $\mathbf{K}_{I}(n)$ is $r$-halving as well: We can set half(c) := $\left(\text { half }\left(\mathfrak{c}_{i}\right)\right)_{i \in I}$. This satisfies Definition 3.1] of halving: Assume that $\mathfrak{D} \in \boldsymbol{\Sigma}(\mathrm{half}(\mathfrak{c}))$ and $\operatorname{nor}(\mathfrak{D})>0$. So $\mathfrak{D}=\left(\mathfrak{D}_{i}\right)_{i \in I}, \mathfrak{D}_{i} \in \Sigma\left(\mathfrak{c}_{i}\right)$, and nor $\left(\mathfrak{D}_{i}\right)>0$ for all $i \in I$. We can un-halve each $\mathfrak{D}_{i}$ to some $\mathfrak{D}_{i}^{\prime}$, and set $\mathfrak{D}^{\prime}=\left(\mathfrak{D}_{i}^{\prime}\right)_{i \in I}$. Then $\mathfrak{D}^{\prime}$ is as required.

However, $\mathbf{K}_{I}$ will not satisfy bigness, since a function $F: \prod_{i \in I} \operatorname{val}\left(\mathfrak{c}_{i}\right) \rightarrow 2$ can generally not be written as a product of functions $F_{i}: \operatorname{val}\left(\mathfrak{c}_{i}\right) \rightarrow 2$. So to handle bigness we have to introduce a new notion:

Definition 4.1. Let $0<r \leq 1, B, K, n>0$.

- $\mathfrak{c}$ is hereditarily $(B, r)$-big, if every $\mathfrak{D} \in \boldsymbol{\Sigma}(\mathfrak{c})$ with nor $(\mathfrak{D})>1$ is $(B, r)$-big.

- $\mathfrak{c}$ is $(K, n, r)$-decisive, if there are $\mathfrak{D}^{-}, \mathfrak{D}^{+} \in \Sigma(\mathfrak{c})$ such that $\operatorname{nor}\left(\mathfrak{D}^{-}\right), \operatorname{nor}\left(\mathfrak{D}^{+}\right) \geq \operatorname{nor}(\mathfrak{c})-r,\left|\operatorname{val}\left(\mathfrak{D}^{-}\right)\right| \leq K$ and $\mathfrak{D}^{+}$is hereditarily $\left(2^{K^{n}}, r\right)$-big. $\mathrm{D}^{-}$is called a $K$-small successor, and $\mathrm{D}^{+}$a $K$-big successor of $\mathrm{c}$.

- $\mathfrak{c}$ is $(n, r)$-decisive if $\mathfrak{c}$ is $(K, n, r)$-decisive for some $K$.

- $\mathbf{K}(n)$ is $(n, r)$-decisive if every $\mathfrak{c} \in \mathbf{K}(n)$ with $\operatorname{nor}(\mathfrak{c})>1$ is $(n, r)$-decisive.

An example for decisive, halving creatures can be found in Section 6 .

Lemma 4.2. (1) If $\mathfrak{c}$ is $(n, r)$-decisive (i.e. $\mathrm{c}$ is $\left(K_{0}, n, r\right)$-decisive for some $\left.K_{0}\right)$, then for every $K \in \omega$ there is either a K-big successor or a $K$-small successor of c.

(2) If $\mathrm{c}$ is $(K, n, r)$-decisive and hereditarily $(B, r)$-big, and if $\operatorname{nor}(\mathfrak{c})>1+r$, then $B<K$.

(3) Assume that $\mathbf{K}(n)$ is $(n, r)$-decisive and $(B, r)$-big for some $B \geq 1$, that $\delta \in \omega$ and that $\operatorname{nor}(\mathfrak{c})>1+\delta \cdot r$. Then there is a hereditarily $(\operatorname{EXP}(B, n, \delta), r)$-big $\mathfrak{D} \in \mathbf{\Sigma}(\mathfrak{c})$ such that $\operatorname{nor}(\mathrm{D}) \geq \operatorname{nor}(\mathfrak{c})-\delta \cdot r$, where $\operatorname{EXP}(B, n, 0)=B$ and $\operatorname{EXP}(B, n, m+1)=$ $2^{\operatorname{EXP}(B, n, m)^{n}}$.

(4) In particular, if $\mathbf{K}(n)$ is $(n, r)$-decisive and $\operatorname{nor}(\mathfrak{c})>1+r$, then there is a hereditarily $(2, r)$-big $\mathfrak{D} \in \mathbf{\Sigma}(\mathfrak{c})$ such that $\operatorname{nor}(\mathfrak{D}) \geq \operatorname{nor}(\mathfrak{c})-r$.

(5) We can avoid small sets without decreasing the norm too much: Assume that $\mathbf{K}(n)$ is $(n, r)$-decisive and $(B, r)$-big for some $B \geq 1$, that $\delta \in \omega$ and that $\operatorname{nor}(\mathrm{c})>$ $1+(\delta+1) \cdot r$. If $X \subseteq \operatorname{val}(\mathfrak{c})$ has size less than $\operatorname{EXP}(B, n, \delta)$, then there is $a \mathfrak{D} \in \mathbf{\Sigma}(\mathfrak{c})$ such that $\operatorname{nor}(\mathrm{D}) \geq \operatorname{nor}(\mathfrak{c})-(\delta+1) \cdot r$ and $\operatorname{val}(\mathrm{D})$ is disjoint to $X$.

Proof. (1): If $K \leq K_{0}$, use $\mathfrak{D}^{-}$, otherwise use $\mathfrak{D}^{+}$. (2): The $K$-small successor $\mathfrak{D}^{-}$is $B$-big, and $\left|\operatorname{val}\left(\mathfrak{D}^{-}\right)\right|<K$. Now use (3.1). (3): Set $\mathfrak{b}_{0}^{+}=\mathfrak{c}$. Assume that $\mathfrak{b}_{i}^{+}$is defined and has norm bigger than 1 . So $\mathfrak{D}_{i}^{+}$is decisive, i.e. there is a $K_{i}$ and a $K_{i}$-small successor $\mathfrak{D}_{i+1}^{-}$and a $K_{i}$-big successor $\mathfrak{b}_{i+1}^{+}$. According to (2), $K_{0}>B$, and $K_{i+1}>2^{K_{i}^{n}} \geq \operatorname{EXP}(B, n, i+1)$. In particular, $\mathrm{D}_{\delta}^{+}$is hereditarily $\operatorname{EXP}(B, n, \delta)$-big. (4): Every creature is $(1, r)$-big. (5) follows

\footnotetext{
${ }^{10}$ So an $n$-creature "lives" on the product $\prod_{i \in I}\left[\mathbf{F}_{i}(n), \mathbf{F}_{i}(n+1)-1\right]$. This does not fit our restrictive framework, so we could just "linearize" the product. Assume $I \in \omega$, i.e. $I=\{0, \ldots, I-1\}$. Set $\mathbf{F}_{I}(n):=\sum_{i \in I} \mathbf{F}_{i}(n)$ and write it in the following way:

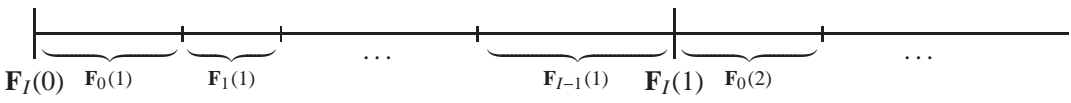

Now it should be clear how to formally define $\mathbf{H}_{I}, \mathbf{K}_{I}, \mathbf{\Sigma}_{I}$ etc.
} 
from (3): First get a $(\operatorname{EXP}(B, n, \delta), r)$-big creature $\mathrm{D}_{0}$, then use the function $F$ that maps val $\mathrm{D}_{0}$ to $X \cup\{$ NotInX $\}$ and thin out $\mathrm{D}_{0}$ to get an $F$-homogeneous $\triangleright$.

We now show by induction on $k$ : If the $n$-creatures are $(k, r)$-decisive, then we can generalize bigness to $k$-tuples.

Lemma 4.3. Assume that $k, m, t \geq 1,0<r \leq 1, \mathfrak{c}_{0}, \ldots, \mathfrak{c}_{k-1} \in \mathbf{K}(n)$ and $F$ satisfy the following:

- $\operatorname{nor}\left(\mathfrak{c}_{i}\right)>1+r \cdot(k-1)$,

- $\mathbf{K}(n)$ is $(k, r)$-decisive and each $c_{i}$ is hereditarily $\left(2^{m^{t}}, r\right)$-big, and

- $F$ is a function from $\prod_{i \in k} \operatorname{val}\left(\mathfrak{c}_{i}\right)$ to $2^{m^{t}}$.

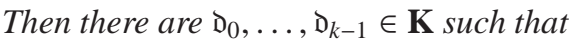

- $\mathrm{D}_{i} \in \boldsymbol{\Sigma}\left(\mathfrak{c}_{i}\right)$,

- $\operatorname{nor}\left(\mathfrak{D}_{i}\right) \geq \operatorname{nor}\left(\mathfrak{c}_{i}\right)-r \cdot k$, and

- $F \uparrow \prod_{i \in k} \operatorname{val}\left(\mathrm{D}_{i}\right)$ is constant.

Proof. The case $k=1$ follows directly from Definition 3.1 of $\left(2^{m^{t}}, r\right)$-big (decisive is not needed). So assume the lemma holds for $k$, and let us investigate the case $k+1$.

$\mathfrak{c}_{k}$ is $(k+1, r)$-decisive, i.e. there is an $M$ such that $\mathfrak{c}_{k}$ is $(M, k+1, r)$-decisive. So 4.2(2) implies

$$
M>2^{m^{t}} .
$$

According to $4.2(1)$, for each $\mathfrak{c}_{i}(i<k)$ we can pick some $\mathfrak{D}_{i}$ that is either an $M$-small successor or an $M$-big successor of $\mathfrak{c}_{i}$ (since each $\mathfrak{c}_{i}$ is $(k+1, r)$-decisive). If $\mathrm{D}_{0}$ is $M$-small, then we let $\mathfrak{D}_{k}$ be the $M$-big successor of $\mathfrak{c}_{k}$, otherwise the $M$-small one. (For $\mathfrak{c}_{k}$ we have both options, since $\mathfrak{c}_{k}$ is $(M, k+1, r)$-decisive.)

This gives a sequence $\left(\mathfrak{b}_{i}\right)_{i \in k+1}$ satisfying $\mathfrak{b}_{i} \in \boldsymbol{\Sigma}\left(\mathfrak{c}_{i}\right)$ and nor $\left(\mathfrak{b}_{i}\right) \geq \operatorname{nor}\left(\mathfrak{c}_{i}\right)-r$. Set $S:=\left\{i \in k+1: \triangleright_{i}\right.$ is $M$-small $\}$, and $L:=(k+1) \backslash S$. So $\{L, S\}$ is a non-trivial partition of $k+1$, since 0 and $k$ are in different sets. If $i \in S$, then $\left|\operatorname{val}\left(\triangleright_{i}\right)\right|<M$, if $i \in L$ then $\triangleright_{i}$ is hereditarily $2^{M^{k+1}}$-big.

Set $Y:=\prod_{i \in S} \operatorname{val}\left(\mathfrak{D}_{i}\right) .|Y| \leq M^{|S|}$. So we can write $Y$ as $\left\{y_{1}, \ldots, y_{\left.M^{|s|}\right\}}\right.$.

Define $F^{*}$ on $\prod_{i \in L} \operatorname{val}\left(\grave{D}_{i}\right)$ by

$$
F^{*}(x):=\left(F\left(x^{\frown} y_{1}\right), \ldots, F\left(x^{\frown} y_{M^{|s|}}\right)\right) .
$$

So (using (4.1) for the last inequality) we get:

$$
\left|\operatorname{image}\left(F^{*}\right)\right| \leq|\operatorname{image}(F)|^{M^{|S|}} \leq 2^{m^{t} M^{|S|}}<2^{M^{|S|+1}} .
$$

For $i \in L, \mathrm{D}_{i}$ is hereditarily $2^{M^{k+1}}$-big and therefore $2^{M^{|S|+1}}$-big, and $|L| \leq k$. Therefore we can apply the induction hypothesis to $k^{\prime}:=|L|, m^{\prime}:=M, t^{\prime}:=|S|+1, F^{\prime}:=F^{*}$ and $c_{i}^{\prime}:=\mathfrak{D}_{i}$ for $i \in L$. This gives us $\left(\mathfrak{D}_{i}^{\prime}\right)_{i \in L}$ such that

- $\mathfrak{D}_{i}^{\prime} \in \boldsymbol{\Sigma}\left(\mathfrak{D}_{i}\right) \subseteq \Sigma\left(\mathfrak{c}_{i}\right)$,

- $\operatorname{nor}\left(\mathfrak{D}_{i}^{\prime}\right) \geq \operatorname{nor}\left(\mathfrak{D}_{i}\right)-r \cdot k^{\prime} \geq \operatorname{nor}\left(\mathfrak{c}_{i}\right)-r(k+1)$, and

- $F^{*} \uparrow \prod_{i \in L} \operatorname{val}\left(\grave{\triangleright}_{i}^{\prime}\right)$ is constant, say $\left(F^{* *}\left(y_{1}\right), \ldots, F^{* *}\left(y_{M^{|S|}}\right)\right)$.

$F^{* *}$ is a function from $Y=\prod_{i \in S} \operatorname{val}\left(\mathrm{D}_{i}\right)$ to $2^{m^{t}}$. Now we apply the induction hypothesis again, this time to $k^{\prime \prime}:=|S|<k+1, m^{\prime \prime}:=m, t^{\prime \prime}=t, F^{\prime \prime}:=F^{* *}$, and $c_{i}^{\prime \prime}:=\mathfrak{D}_{i}$ for $i \in S$. This gives us $\left(\widehat{D}_{i}^{\prime}\right)_{i \in S}$ such that

- $\mathfrak{D}_{i}^{\prime} \in \boldsymbol{\Sigma}\left(\mathfrak{D}_{i}\right) \subseteq \boldsymbol{\Sigma}\left(\mathfrak{c}_{i}\right)$,

- $\operatorname{nor}\left(\triangleright_{i}^{\prime}\right) \geq \operatorname{nor}\left(\mathrm{D}_{i}\right)-r \cdot k^{\prime \prime} \geq \operatorname{nor}\left(\mathfrak{c}_{i}\right)-r(k+1)$, and

- $F^{* *} \uparrow \prod_{i \in S} \operatorname{val}\left(\left(_{i}^{\prime}\right)\right.$ is constant. 
Then $\left(\grave{D}_{i}^{\prime}\right)_{i \leq k}$ is as required.

According to 4.2 3 ), we can increase the hereditary bigness by decreasing the norm. So we get (again setting $\operatorname{EXP}(B, n, 0)=B$ and $\left.\operatorname{EXP}(B, n, m+1)=2^{\operatorname{EXP}(B, n, m)^{n}}\right)$ :

Corollary 4.4. Fix $\delta \geq 1$. Assume that $k \geq 1,0<r \leq 1, \mathbf{K}(n)$ is $(k, r)$-decisive and $(B, r)$ big, $\operatorname{nor}\left(\mathfrak{c}_{i}\right)>1+r \cdot(\delta+k-1)$ for $0 \leq i<k$ and $F: \prod_{i \in k} \operatorname{val}\left(\mathfrak{c}_{i}\right) \rightarrow \operatorname{EXP}(B, k, \delta)$. Then there are $\mathfrak{b}_{i} \in \boldsymbol{\Sigma}\left(\mathfrak{c}_{i}\right)$ with $F$-homogeneous product such that $\operatorname{nor}\left(\mathfrak{b}_{i}\right) \geq \operatorname{nor}\left(\mathfrak{c}_{i}\right)-r \cdot(\delta+k)$.

Proof. By first decreasing the norms by at most $\delta \cdot r$, we can assume that each $c_{i}$ is hereditary $\operatorname{EXP}(B, k, \delta)$-big. Now use Lemma4.3 (Note that $\operatorname{EXP}(B, n, \delta)$ is of the form $2^{m^{t}}$ for some $m$ and $t$.)

Every creature is $(1, r)$-big, and $\operatorname{EXP}(1, n, 1)=2$. So we get for $\delta=1$ :

Corollary 4.5. Assume that $k \geq 1,0<r \leq 1, \mathbf{K}(n)$ is $(k, r)$-decisive, $\operatorname{nor}\left(\mathfrak{c}_{i}\right)>1+r \cdot k$ for $0 \leq i<k$ and $F: \prod_{i \in k} \operatorname{val}\left(\mathfrak{c}_{i}\right) \rightarrow 2$. Then there are $F$-homogeneous $\triangleright_{i} \in \Sigma\left(\mathfrak{c}_{i}\right)$ such that $\operatorname{nor}\left(\mathfrak{D}_{i}\right) \geq \operatorname{nor}\left(\mathfrak{c}_{i}\right)-r \cdot(k+1)$.

In other words: If we assume that $\mathbf{K}_{i}(n)$ is $(|I|, r)$-decisive for all $i \in I$, then every $\mathfrak{c} \in \mathbf{K}_{I}(n)$ with $\operatorname{nor}(\mathfrak{c})>1+r \cdot|I|$ is $(2, r \cdot(|I|+1))$-big.

In particular, we get pure decision for the finite product:

Corollary 4.6. Set $\varphi(<n):=\prod_{i \in I} \prod_{m<\mathbf{F}_{i}(n)} \mathbf{H}_{i}(m)$, and $r(n):=1 /(n \varphi(<n))$. Assume that for all $i \in I$ and $n \in \omega, \mathbf{K}_{i}(n)$ is $(|I|, r(n))$-decisive and $r(n)$-halving. Then $\prod_{i \in I} \mathbb{Q}_{\infty}^{*}\left(\mathbf{K}_{i}, \boldsymbol{\Sigma}_{i}\right)$ is $\omega^{\omega}$-bounding and proper and preserves the size of the continuum. Under $C H, \prod_{i \in I} \mathbb{Q}_{\infty}^{*}\left(\mathbf{K}_{i}, \boldsymbol{\Sigma}_{i}\right)$ is $\boldsymbol{\aleph}_{2}$-cc and preserves all cardinals.

Proof. $\prod_{i \in I} \mathbb{Q}_{\infty}^{*}\left(\mathbf{K}_{i}, \boldsymbol{\Sigma}_{i}\right)=\mathbb{Q}_{\infty}^{*}\left(\mathbf{K}_{I}, \boldsymbol{\Sigma}_{I}\right) . \quad \mathbf{K}_{I}(n)$ is $r(n)$-halving and $(2, r(n) \cdot(|I|+1))$-big according to Corollary 4.5 (Actually we get bigness only for creatures with norm bigger than $1+r \cdot|I|$ instead of 1.) Now use Theorem 3.2 and the Note following it. Note that $\prod_{i \in I} \mathbb{Q}_{\infty}^{*}\left(\mathbf{K}_{i}, \boldsymbol{\Sigma}_{i}\right)$ has size $2^{\mathbf{\aleph}_{0}}$.

Remark: Decisiveness is quite costly: To be able to apply the last corollary, we will have to make the $n$-th level much larger than levels before, i.e.

$$
\prod_{F_{i}(n) \leq m<F_{i}(n+1)} \mathbf{H}_{i}(m) \gg \prod_{j \in I} \prod_{m<F_{i}(n)} \mathbf{H}_{j}(m)
$$

for all $i \in I$. In our application this will have the effect that we can separate $(f, g)$ and $\left(f^{\prime}, g^{\prime}\right)$ only if their growth rates are considerably different. It is very likely that with a more careful and technically more complicated analysis one can construct forcings that can separate cardinal invariants for pairs that are not so far apart, but this would need other concepts than decisiveness.

\section{A VARIANT OF THE COUNTABLE SUPPORT PRODUCT}

We now define $P$, a variant of the countable support product of lim-inf creature forcings. We want to end up with a forcing notion that also satisfies fusion, pure decision and $\boldsymbol{\aleph}_{2}$-cc (under $\mathrm{CH}$ ). This will give preservation of all cardinals. We will also need rapid reading of names.

Let $I$ be the index set of the product. We will use $\alpha$ and $\beta$ for elements of $I$.

Assumption 5.1. Fix a set $I$ and for every $\alpha \in I$, a creating pair $\left(\mathbf{K}_{\alpha}, \boldsymbol{\Sigma}_{\alpha}\right)$. We assume that for each $n$ there is an upper bound $m(n)$ for $\left|\prod_{\mathbf{F}_{\alpha}(n) \leq i<\mathbf{F}_{\alpha}(n+1)} \mathbf{H}_{\alpha}(i)\right|$, and set $\varphi(=n):=m(n)^{n}$, $\varphi(\leq n):=\prod_{m \leq n} \varphi(=m)$ and $\varphi(<n):=\prod_{m<n} \varphi(=m)$. 
We define the set $P$ in the following way:

Definition 5.2. A condition $p$ in $P$ consists of a countable subset $\operatorname{dom}(p)$ of $I$, of objects $p(\alpha, n)$ for $\alpha \in \operatorname{dom}(p), n \in \omega$, and of a function $\operatorname{trnklh}(p): \operatorname{dom}(p) \rightarrow \omega$ satisfying the following $(\alpha \in \operatorname{dom}(p))$ :

- If $n<\operatorname{trnklh}(p, \alpha)$, then $p(\alpha, n) \in \prod_{\mathbf{F}_{\alpha}(n) \leq i<\mathbf{F}_{\alpha}(n+1)}\left(\mathbf{H}_{\alpha}(i)\right)$.

$\bigcup_{n<\operatorname{trnklh}(p)} p(\alpha, n)$ is called trunk of $p$ at $\alpha$.

- If $n \geq \operatorname{trnklh}(p, \alpha)$, then $p(\alpha, n) \in \mathbf{K}_{\alpha}(n)$ and $\operatorname{nor}(p(\alpha, n))>0$.

- $|\operatorname{supp}(p, n)|<n$ for all $n>0$, where we set

$$
\operatorname{supp}(p, n):=\{\alpha \in \operatorname{dom}(p): \operatorname{trnklh}(p, \alpha) \leq n\} .
$$

- Moreover, $\lim _{n \rightarrow \infty}(|\operatorname{supp}(p, n)| / n)=0$.

- $\lim _{n \rightarrow \infty}(\min (\{\operatorname{nor}(p(\alpha, n)): \alpha \in \operatorname{supp}(p, n)\}))=\infty$.

So in particular, for $\alpha \in \operatorname{dom}(p)$ the sequence $(p(\alpha, n))_{n \in \omega}$ is in $\mathbb{Q}_{\infty}^{*}\left(\mathbf{K}_{\alpha}, \boldsymbol{\Sigma}_{\alpha}\right)$.

Note that now there is an essential difference between a part $t$ of the trunk and creature $\mathfrak{c}$ with $\operatorname{val}(\mathfrak{c})=\{t\}$ : The trunks do not prevent the minimum of the norms at height $h$ to be large.

Remarks. - For the proof of Theorem 1 we will additionally fix a function trnklh $^{\text {min }}$ : $I \rightarrow \omega$ and add the following requirement to the definition of $P$ :

$$
\operatorname{trnklh}(p, \alpha) \geq \operatorname{trnklh}^{\min }(\alpha) .
$$

This does not change any of the following properties of $P$ (or their proofs).

- For the proof of Theorem 2, we will define the forcing $R$ so that a condition $p$ picks for each $\alpha \in \operatorname{dom}(p)$ one of several possibilities for a creating pair $\left(\mathbf{K}_{\alpha}, \boldsymbol{\Sigma}_{\alpha}\right)$. It turns out that this does not change anything either, apart from the fact that $R_{\epsilon}$ is not a complete subforcing of $R$ any more, i.e. Lemma 5.5 fails. Lemma 5.4 still holds but needs a new proof. The rest of the proofs still work without changes.

As outlined, we have to modify the order usually used in the product:

Definition 5.3. $q \leq p$ if

- $\operatorname{dom}(q) \supseteq \operatorname{dom}(p)$,

- if $\alpha \in \operatorname{dom}(p)$ and $n \in \omega$, then $q(\alpha, n) \in \mathbf{\Sigma}(p(\alpha, n))$,

- $\operatorname{trnklh}(q, \alpha)=\operatorname{trnklh}(p, \alpha)$ for all but finitely many $\alpha \in \operatorname{dom}(p)$.

Note that $q \leq p$ implies that then $\operatorname{trnklh}(q, \alpha) \geq \operatorname{trnklh}(p, \alpha)$ for all $\alpha \in \operatorname{dom}(p)$.

Figure 3 shows one way to visualize $q \leq p$.

If $I$ is finite then $P$ is just the product $\prod_{\alpha \in I} \mathbb{Q}_{\infty}^{*}\left(\mathbf{K}_{\alpha}, \mathbf{\Sigma}_{\alpha}\right)$.

For every $\alpha \in I, P$ adds a generic real $\eta_{\alpha}$, defined as the union of the trunks of $p$ at $\alpha$ for $p$ in the generic filter. It is easy to see that $\eta_{\alpha}$ is forced to be different from $\eta_{\beta}$ for $\alpha \neq \beta$. Once again, the sequence $\left(\eta_{\alpha}\right)_{\alpha \in I}$ does not determine the generic filter.

Conditions with disjoint domains are compatible:

Lemma 5.4. ( $\mathrm{CH}) \mathrm{P}$ is $\boldsymbol{\aleph}_{2}-c c$.

Proof. Assume towards a contradiction that $A$ is an antichain of size $\boldsymbol{\aleph}_{2}$. Without loss of generality, $(\operatorname{dom}(a))_{a \in A}$ forms a $\Delta$-system with root $u$. There are at most $2^{\aleph_{0}}$ many possibilities for $a \uparrow u$, so without loss of generality, $p \uparrow u=q \uparrow u$ for all $p, q \in A$. Then $p$ and $q$ are compatible: The function $x(n)=|\operatorname{supp}(p, n) \cup \operatorname{supp}(q, n)| / n$ converges to 0 . So there is an $h$ such that $x(m)<1$ for all $m \geq h$. Construct $r$ from $p \cup q$ by enlarging the (finitely many) trunks at $\operatorname{supp}(q, h) \cup \operatorname{supp}(p, h)$ to height $h$. Then $r \in P$ and $r \leq p, q$. 


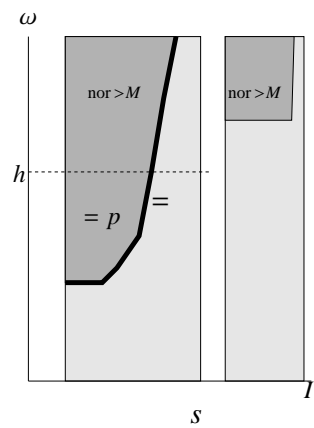

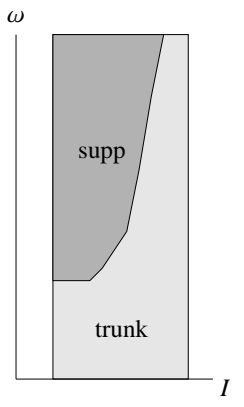

$p$

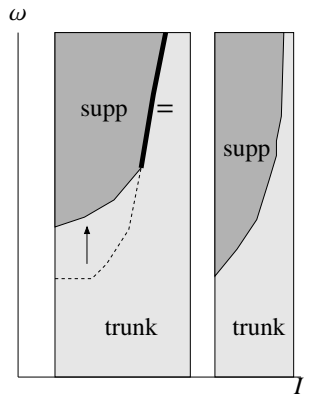

$q$

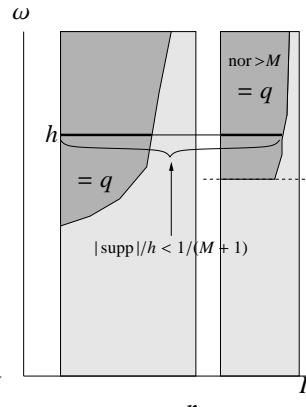

$r$

FIGURE 3. $q \leq p, s \leq_{M} p, r \leq_{M}^{\text {new }} p$.

Lemma 5.5. If $J \subseteq I$, then $P_{J}=\{p \in P: \operatorname{dom}(p) \subseteq J\}$ is a complete subforcing of $P$.

Proof. If $p \in P$, then $p \uparrow J \in P_{J}$, and $q \leq_{P} p$ implies $q \uparrow J \leq_{P_{J}} p \uparrow J$. So if $p \perp_{P_{J}} q$, then $p \perp_{P} q$. Also, $p\left\lceil J\right.$ is a reduction of $p$ : If $q \leq_{P_{J}} p \uparrow J$, then we can again enlarge finitely many stems of $q \cup p \uparrow(I \backslash J)$ to get a condition $r \in P$ which is stronger than both $p$ and $q$.

Definition 5.6. - $\operatorname{val}^{\Pi}(p,<n):=\prod_{\alpha \in \operatorname{dom}(p)} \prod_{m<n} \operatorname{val}(p(\alpha, m))$. The size of this set is at most $\varphi(<n) \cdot \operatorname{val}^{\Pi}(p, \leq n):=\operatorname{val}^{\Pi}(p,<(n+1))$.

- If $w \subseteq \operatorname{dom}(p)$ and $t \in \prod_{\alpha \in w} \prod_{0 \leq m<\mathbf{F}_{\alpha}(n)} \mathbf{H}_{\alpha}(m)$, then $p \wedge t$ is defined by

$$
(p \wedge t)(\alpha, m)= \begin{cases}t_{\alpha} \uparrow\left[\mathbf{F}_{\alpha}(m), \mathbf{F}_{\alpha}(m+1)-1\right] & \text { if } m<n \text { and } \alpha \in w, \\ p(\alpha, m) & \text { otherwise. }\end{cases}
$$

So $p \wedge t \in P$, and if $t \in \operatorname{val}^{\Pi}(p,<n)$, then $p \wedge t \leq p$.

- If $\tau$ is a name of an ordinal, then $p<n$-decides $\tau$, if $p \wedge t$ decides $\tau$ for all $t \in$ $\operatorname{val}^{\Pi}(p,<n) . p$ essentially decides $\underset{\sim}{\tau}$, if $p<n$-decides $\underset{\sim}{\tau}$ for some $n$.

As in the one-dimensional case we get:

Facts 5.7. (1) $\left\{p \wedge t: t \in \mathrm{val}^{\Pi}(p,<n)\right\}$ is predense under $p$ (for $p \in P$ and $n \in \omega$ ).

(2) $p \wedge t \Vdash \varphi$ iff $p \Vdash\left[(\forall \alpha \in \operatorname{dom}(t)) t(\alpha)<\eta_{\alpha} \rightarrow \varphi\right]$.

(3) Assume that $q^{\prime}$ is the result of replacing finitely many creatures $c$ of $q$ by creatures D with $\operatorname{val}(\mathfrak{D}) \subseteq \operatorname{val}(\mathfrak{c})$. Then $q^{\prime} \leq \leq^{*} \underline{11}$

(4) If $q \leq p$ and $t \in \operatorname{val}^{\Pi}(q,<n)$, then $t$ restricted to the domain of $p$ is in $\operatorname{val}^{\Pi}(p,<n)$ !12

(5) If $q \leq p, t \in \operatorname{val}^{\Pi}(q,<n)$, and $s$ is the corresponding element in $\operatorname{val}^{\Pi}(p,<n)$, then $q \wedge t \leq s \wedge p$.

(6) If $q^{\prime} \leq q$ and $q$ essentially decides $\underset{\sim}{\tau}$, then $q^{\prime}$ essentially decides $\tau$.

(7) If $q \wedge t$ essentially decides $\mathcal{\sim}$ for each $t \in \operatorname{val}^{\Pi}(q,<n)$, then $q$ essentially decides $\mathcal{\tau}$.

Recall that $\varphi(<n)$ is an upper bound for the number of possible sequences of trunks of height $n$ (cf.5.1).

\footnotetext{
${ }^{11}$ In other words: Assume that $q, q^{\prime} \in P, h \in \omega$, $\operatorname{dom}\left(q^{\prime}\right)=\operatorname{dom}(q), q(\alpha, m)=q^{\prime}(\alpha, m)$ for all $m \geq h$ and $\alpha \in \operatorname{dom}(q)$, and $\operatorname{val}\left(q_{\alpha}^{\prime}(m)\right) \subseteq \operatorname{val}\left(q_{\alpha}(m)\right)$ for all $m<h$ and $\alpha \in \operatorname{dom}(q)$. Then $q^{\prime} \leq^{*} q$.

${ }^{12}$ The same holds for $q \leq^{*} p$, apart from the fact that $\operatorname{dom}(p)$ might not be a subset of $\operatorname{dom}(q)$. (Outside of $\operatorname{dom}(q), p$ could consists of "maximal creatures with no information".)
} 
Theorem 5.8. If $\mathbf{K}_{\alpha}(n)$ is (n, $\left.r(n)\right)$-decisive and $r(n)$-halving for $r(n)=1 /\left(n^{2} \varphi(<n)\right)$ and every $\alpha \in I, n \in \omega$, then $P$ is proper and $\omega^{\omega}$-bounding. Assume $|I| \geq 2$ and set $\lambda=|I|^{\aleph_{0}}$. Then $P$ forces $|I| \leq 2^{\aleph_{0}} \leq \lambda$.

Proof. The proof closely follows the one-dimensional case. We again prove pure decision and fusion, and the rest follows as in the proof of Theorem 3.2 (Note that $|P|=|I|^{\aleph_{0}}$, and that $\eta_{\alpha}$ and $\eta_{\beta}$ are forced to be different for $\alpha \neq \beta$.)

So we have to define $\leq_{M}$ : First we set $r \leq_{M}^{\text {new }} p$, if $r \leq p$, and

- if $n \in \omega$ and $\alpha \in \operatorname{supp}(r, n) \backslash \operatorname{dom}(p)$, then $n>M,|\operatorname{supp}(r, n)| / n \leq 1 /(M+1)$, and $\operatorname{nor}(r(\alpha, n))>M$.

Assume that $M \in \omega$ and $q \leq p$. By extending finitely many trunks in $q$ at positions $\alpha \notin \operatorname{dom}(p)$, we get an $r \leq q$ such that

$$
r \leq_{M}^{\text {new }} p \text { and } r(\alpha, n)=q(\alpha, n) \text { for } \alpha \in \operatorname{dom}(p)
$$

(cf. Figure 3).

$s \leq_{M}^{\text {old }} p$, if $s \leq p$ and there is an $h \geq M$ such that for all $\alpha \in \operatorname{dom}(p)$,

- $\operatorname{trnklh}(s, \alpha)=\operatorname{trnklh}(p, \alpha)$,

- if $n<h$, then $s(\alpha, n)=p(\alpha, n)$,

- if $\alpha \in \operatorname{supp}(p, n)$ and $n \geq h$, then $\operatorname{nor}(s(\alpha, n)) \geq M$.

$r \leq_{M} p$, if $r \leq_{M}^{\text {new }} p$ and $r \leq_{M}^{\text {old }} p$.

By 5.1 we get:

$$
\text { If } q \leq_{M}^{\text {old }} p \text {, then there is an } r \leq q \text { such that } r \leq_{M} p .
$$

\section{$\leq_{n}$ satisfies fusion:}

Assume that $\left(p^{m}\right)_{m \in \omega}$ satisfies $p^{m+1} \leq_{m+1} p^{m}$. Define $q$ by $\operatorname{dom}(q)=\bigcup_{n \in \omega} \operatorname{dom}\left(p^{n}\right)$ and $q_{\alpha}(n)=p_{\alpha}^{M}(n)$, where $M \geq n$ is minimal (or: arbitrary) such that $\alpha \in \operatorname{dom}\left(p^{M}\right)$. Then $q \in P$ : Fix some $k$. Since $p^{k} \in P$, there is an $l$ such that

(5.3) $\operatorname{nor}\left(p^{k}(\alpha, n)\right)>k$ and $\left|\operatorname{supp}\left(p^{k}, n\right)\right| / n<1 /(k+1)$ for all $n>l$ and $\alpha \in \operatorname{supp}\left(p^{k}, n\right)$.

Since $p^{k+1} \leq_{k+1} p^{k}$, 5.3. holds for $p^{k+1}$ as well, and for all $p^{m}$ with $m>k$, and therefore for $q$.

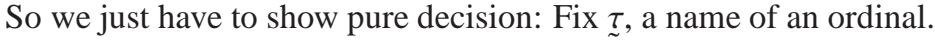

The basic construction $S(p, M)$ :

Let $n$ be the minimal trunk-length of $p$, i.e. $n=\min (\{\operatorname{trnklh}(p, \alpha): \alpha \in \operatorname{dom}(p)\})$. We will now define $S(p, M) \leq p$ for $M \in \omega$.

Enumerate $\operatorname{val}^{\Pi}(p, \leq n)$ as $s^{0}, \ldots, s^{l-1}$. So $l \leq \varphi(=n)$. Set $p^{-1}:=p$. Given $p^{k-1}$, define $p^{k} \leq p^{k-1} \wedge s^{k}$ and $h^{k}$ such that for all $\alpha \in \operatorname{dom}(p)$

- $\operatorname{trnklh}\left(p^{k}, \alpha\right)=\operatorname{trnklh}\left(p^{k-1} \wedge s^{k}, \alpha\right)=\max (n+1, \operatorname{trnklh}(p, \alpha))$,

- if $n<m<h^{k}$, then $\operatorname{nor}\left(p^{k}(\alpha, m)\right) \geq \operatorname{nor}\left(p^{k-1}(\alpha, m)\right)-r(m)$,

- if $m \geq h^{k}$, then $\operatorname{nor}\left(p^{k}(\alpha, m)\right) \geq M$,

and such that additionally one of the following two cases holds:

dec: $p^{k}$ essentially decides $\tau$, or

half: it is not possible to satisfy "dec" (for any choice of $h^{k}$ ), and $\operatorname{dom}\left(p^{k}\right)=\operatorname{dom}\left(p^{k-1}\right)$ and $p^{k}(\alpha, m)=\operatorname{half}\left(p^{k-1}(\alpha, m)\right)$ for all $m>n$ and $\alpha \in \operatorname{supp}\left(p^{k-1}, m\right)$.

\footnotetext{
${ }^{13}$ we do not require anything for $\alpha \in \operatorname{dom}\left(p^{k}\right) \backslash \operatorname{dom}(p)$
} 
So we first try to find a $p^{k}$ satisfying "dec" (possibly with larger domain); if we fail we just halve each $p^{k-1}(\alpha, m)$.

We construct $p^{k}$ for each $0 \leq k<l$. This gives a function

$$
F: \prod_{\alpha \in \operatorname{supp}(p, n)} \operatorname{val}(p(\alpha, n)) \rightarrow\{\mathrm{dec}, \text { half }\} .
$$

Each $\mathbf{K}_{\alpha}(n)$ is $(n, r(n))$-decisive, and $|\operatorname{supp}(p, n)|<n$. So according to Corollary 4.5 (for $k=n-1)$ there are $\mathrm{D}_{\alpha} \in \mathbf{\Sigma}(p(\alpha, n))($ for $\alpha \in \operatorname{supp}(p, n))$ such that $F \uparrow \prod_{\alpha \in \operatorname{supp}(p, n)} \operatorname{val}\left(\mathrm{D}_{\alpha}\right)$ is constant and $\operatorname{nor}\left(\mathrm{D}_{\alpha}\right) \geq \operatorname{nor}(p(\alpha, n))-n \cdot r(n)$.

For this construction to work, we have to assume that the norms of all the creatures involved are big enough (so that we can apply bigness and halving). If this is not the case, $S(p, M)$ is undefined. Otherwise, we set $\operatorname{dom}(S(p, M))=\operatorname{dom}\left(p^{l-1}\right)$ and for $\alpha \in$ $\operatorname{dom}(S(p, M))$

$$
S(p, M)(\alpha, m)= \begin{cases}p(\alpha, m) & \text { if } m<n \text { and } \alpha \in \operatorname{dom}(p), \\ \mathrm{D}_{\alpha} & \text { if } m=n \text { and } \alpha \in \operatorname{dom}(p), \\ p^{l-1}(\alpha, m) & \text { otherwise. }\end{cases}
$$

We call $q=S(p, M)$ halving, if the constant value of $F$ is "half".

If $q$ is not halving, then $q$ essentially decides $\tau$ : If $t \in \operatorname{val}^{\Pi}(q, \leq n)$, then $t$ restricted to $\operatorname{dom}(p)$ is in $\operatorname{val}^{\Pi}(p, \leq n)$, i.e. it is some $s^{k}$. Then $q \wedge t \leq q \wedge s^{k}$, and $q \wedge s^{k}$ is stronger than $p^{k}$, which essentially decides $\tau$. Now use Facts $5.7(6,7)$.

Some properties of $S(p, \tilde{M})$ :

If $q=S(p, M)$ is defined and $n$ the minimal trunk-length of $p$, then:

$$
\begin{aligned}
& \operatorname{nor}(q(\alpha, n)) \geq \operatorname{nor}(p(\alpha, n))-n \cdot r(n) \text { for } \alpha \in \operatorname{supp}(p, n) \text {. } \\
& \operatorname{nor}(q(\alpha, m)) \geq \min (M, \operatorname{nor}(p(\alpha, m)))-\varphi(=n) \cdot r(m) \text { for all } m>n \text { and } \\
& \alpha \in \operatorname{supp}(p, m) \text {. } \\
& \text { If } q \text { is halving, then there is no } q^{\prime} \leq q \text { essentially deciding } \tau \text { such that } \\
& \operatorname{trnklh}\left(q^{\prime}, \alpha\right)=\max (n+1, \operatorname{trnklh}(p, \alpha)) \text { for all } \alpha \in \operatorname{dom}(p) .
\end{aligned}
$$

To see (5.6), assume that $q^{\prime}$ is a counterexample and that $h$ is such that nor $\left(q^{\prime}(\alpha, m)\right)>M$ for all $m>h$ and $\alpha \in \operatorname{supp}\left(q^{\prime}, m\right)$. Let $t$ be in $\operatorname{val}^{\Pi}\left(q^{\prime}, \leq n\right)$. $t$ restricted to $\operatorname{dom}(p)$ is $s^{k}$ for some $k<l$. We know that $p^{k}$ was constructed by halving each creature of $p^{k-1} \wedge s^{k}$ and that $q^{\prime} \leq p^{k}$. We now define $r$ : $\operatorname{Set} \operatorname{dom}(r)=\operatorname{dom}\left(q^{\prime}\right)$. If $m \leq h$ and $\alpha \in \operatorname{supp}(p, m)$, we un-halve $q^{\prime}(\alpha, m)$ to some $\delta(\alpha, m)$ and set $r(\alpha, m)=\delta(\alpha, m)$. Otherwise we set $r(\alpha, m)=$ $q^{\prime}(\alpha, m)$. According to 5.7 $(3,6) r$ essentially decides $\tau$. So we should have chosen $r$ instead of $p^{k}$, a contradiction.

$S(p, M)$ essentially decides:

Assume that $M>3$, and that nor $(p(\alpha, m))>3$ for all $m \in \omega$ and $\alpha \in \operatorname{supp}(p, m)$. We now show that $S(p, M)$ exists and is not halving.

Assume towards a contradiction that $S(p, M)$ is halving. Let $n$ be again the minimal trunk-length of $p$. We set $q^{n-1}=p$. Assume that for $k \geq n-1, q^{k}$ is already defined. We set $M_{k}=M+k+1-n$. (So $M_{n-1}=M$.) We define $q^{k+1}$ the following way: $\operatorname{List}_{\operatorname{val}}{ }^{\mathrm{I}}\left(q^{k}, \leq k\right)$ as $s^{0}, \ldots, s^{l-1}$. So $l \leq \varphi(\leq k)$. Set $r^{-1}:=q^{k}$. Given $r^{i-1}$, set $r^{i}=S\left(r^{i-1} \wedge s^{i}, M_{k}\right)$ (if defined). Define $q^{k+1}$ to be $q^{k}$ up to $k$ and $r^{l-1}$ otherwise, and additionally increase the stems outside $\operatorname{dom}\left(q^{k}\right)$ to satisfy $q^{k+1} \leq_{M_{k}}^{\text {new }} q^{k}$. More formally: We pick some $h>M_{k}, h>k$ such that that $\operatorname{nor}\left(r^{l-1}(\alpha, m)\right)>M_{k}$ and $\left|\operatorname{supp}\left(r^{l-1}, m\right)\right| / m<1 / M_{k}$ for all $m>h$ and $\alpha \in \operatorname{supp}\left(r^{l-1}, m\right)$. For $\alpha \in \operatorname{dom}\left(r^{l-1}\right) \backslash \operatorname{dom}\left(q^{k}\right)$ and $m \leq h$, we pick some $t(\alpha, m) \in \operatorname{val}\left(r^{l-1}(\alpha, m)\right)$. The we 
define $q^{k+1} \operatorname{by} \operatorname{supp}\left(q^{k+1}\right)=\operatorname{supp}\left(r^{l-1}\right)$ and

$$
q^{k+1}(\alpha, m)= \begin{cases}q^{k}(\alpha, m) & \text { if } m \leq k \text { and } \alpha \in \operatorname{dom}\left(q^{k}\right), \\ r^{l-1}(\alpha, m) & \text { if } m>h \text { or if } m>k \text { and } \alpha \in \operatorname{dom}\left(q^{k}\right), \\ t(\alpha, m) & \text { if } m \leq h \text { and } \alpha \notin \operatorname{dom}\left(q^{k}\right) .\end{cases}
$$

Note that $q^{n}$ is just $S(p, M)$ with some increased trunks outside of $\operatorname{dom}(p)$.

$q^{k+1}$ satisfies for $\alpha \in \operatorname{dom}\left(q^{k}\right), \beta \in \operatorname{dom}\left(q^{k+1}\right)$ :

- $q^{k+1}(\alpha, m)=q^{k}(\alpha, m)$ for $m \leq k$.

- $\operatorname{nor}\left(q^{k+1}(\alpha, k+1)\right) \geq \operatorname{nor}\left(q^{k}(\alpha, k+1)\right)-\varphi(\leq k) \cdot(k+1) \cdot r(k+1)$.

- $\operatorname{nor}\left(q^{k+1}(\alpha, m)\right) \geq \min \left(M^{k}, \operatorname{nor}\left(q^{k}(\alpha, m)\right)\right)-\varphi(\leq k+1) \cdot r(m)$ for $m>k+1$.

- $\operatorname{nor}\left(q^{k+1}(\beta, m)\right) \geq M^{k}$ if $\beta \in \operatorname{supp}\left(q^{k+1}, m\right) \backslash \operatorname{dom}\left(q^{k}\right)$.

Iterating this $l$ many times, we get:

$$
\operatorname{nor}\left(q^{k+l}(\alpha, m)\right) \geq \min \left(M^{k}, \operatorname{nor}\left(q^{k}(\alpha, m)\right)\right)-\min (l, m-k) \cdot \varphi(<m) \cdot m \cdot r(m),
$$

so according to the definition of $r(m)$ we get

$$
\operatorname{nor}\left(q^{k+l}(\alpha, m)\right) \geq \min \left(M^{k}, \operatorname{nor}\left(q^{k}(\alpha, m)\right)\right)-1 .
$$

This shows, as in the one-dimensional case, that each $q^{m}$ is defined, and that $q^{\omega}$ is a condition in $P$, where we define $q^{\omega}$ by $\operatorname{dom}\left(q^{\omega}\right)=\bigcup_{k \in \omega} q^{k}$, and $q^{\omega}(\alpha, m)=q^{k}(\alpha, m)$, where $k$ is the minimal (or: some) $k \geq m$ such that $\alpha \in \operatorname{dom}\left(q^{k}\right)$. Just as for (3.18), we can generalize (5.6) by induction and get:

There is no $q^{\prime} \leq q^{k}$ essentially deciding $\tau$ such that

$$
\operatorname{trnklh}\left(q^{\prime}, \alpha\right)=\max \left(k+1, \operatorname{trnklh}\left(q^{k}, \alpha\right)\right) \text { for all } \alpha \in \operatorname{dom}\left(q^{k}\right) \text {. }
$$

But there is a $q^{\prime} \leq q^{\omega}$ deciding $\tau$. This implies that the trunk-lengths of $q^{\prime}$ and of $q^{\omega}$ are the same on almost all elements of the domain of $q^{\omega}$. So by increasing finitely many trunks of $q^{\prime}$, we can assume that $\operatorname{trnklh}\left(q^{\prime}, \alpha\right)=\max \left(k+1, \operatorname{trnklh}\left(q^{\omega}, \alpha\right)\right)$ for some $k$. So $q^{\prime} \leq q^{k}$ decides $\tau$, , a contradiction to $5.9,14$

\section{Pure decision:}

Given $p$ and $M$, we find an $h>M+6$ such that $\operatorname{nor}(p(\alpha, m))>M+6$ for all $m \geq h$ and $\alpha \in \operatorname{supp}(p, m)$. Enumerate val ${ }^{\Pi}(p, \leq h-1)$ as $\left\{s^{1}, \ldots, s^{l}\right\}$. As above, set $p^{0}=p$, $p^{k+1}=S\left(p^{k} \wedge s^{k}, M+6\right)$, and define $q$ by $q(\alpha, m)=p(\alpha, m)$ for $m<h$ and $\alpha \in \operatorname{dom}(p)$, and by $q(\alpha, m)=p^{l-1}(\alpha, m)$ otherwise. Then $q \leq_{M}^{\text {old }} p$ essentially decides $\underset{\sim}{\tau}$, and according to (5.2) we find a $q^{\prime} \leq q$ such that $q \leq_{M} p$.

As already mentioned, only the growth rate of $r(n)$ is relevant. Since we are dealing with decisive creatures, we can increase bigness even exponentially (in $n$ ) while decreasing the norms by a constant factor (cf. Corollary 4.5). We use this for the following version of rapid reading. Again, we set $\operatorname{EXP}(B, n, 0)=B$ and $\operatorname{EXP}(B, n, k+1)=2^{\operatorname{EXP}(B, n, k)^{n}}$; and we define $r, \varphi$ as in the previous theorem.

Theorem 5.9. Assume that

- $\delta \in \omega$,

- $g: \omega \rightarrow \omega$ is monotonously increasing,

- $\mathbf{K}_{\alpha}(n)$ is ( $(g(n), r(n))$-big, $(n, r(n))$-decisive and $r(n)$-halving for all $\alpha \in I, n \in \omega$,

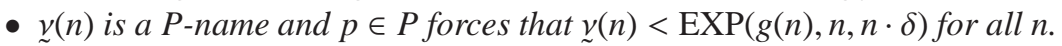

Then there is a $q \leq p$ which $<n$-decides $y(n)$ for all $n$.

\footnotetext{
${ }^{14}$ So this step in the proof is the reason that we had to redefine $\leq$.
} 
Proof. We make the same modification to the previous proof as in the one-dimensional case:

The basic construction $S(p, l, M)$ : We again assume that $n$ is the minimal length of the trunks in $p$, and use the notation $S(p, l, M)$ (for $l \leq n)$ for the same construction as $S(p, M)$,

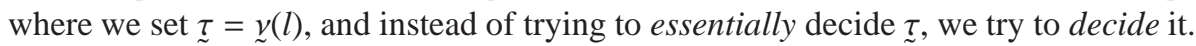

So instead of the two cases "dec" and "half", we get $\operatorname{EXP}(g(n), n, n \cdot \delta)+1$ many cases: one for each potential value of $\mathcal{z}(n)$, and (if none of these cases can be satisfied) "half". So the number of possible cases is less than $\operatorname{EXP}(g(n), n, n \cdot(\delta+1))$. We use Corollary 4.4 to find successors $q(\alpha, m)$ of $p(\alpha, m)$ with $F$-homogeneous product. This decreases the norm by at most $r(n) \cdot(n(\delta+1)+n)$, i.e. by $n \cdot(\delta+2) \cdot r(n)$.

Some properties of $S(p, l, M)$ : So instead of (5.4) we get

$$
\operatorname{nor}(q(\alpha, n)) \geq \operatorname{nor}(p(\alpha, n))-n(\delta+2) \cdot r(n) \text { for } \alpha \in \operatorname{supp}(p, n) .
$$

There is no change to (5.5), and in (5.6) we replace "essentially deciding $\underset{\sim}{ }$ " with "deciding $y(l) "$

$S(p, l, M)$ decides: We again construct $q^{k}$, each time trying to decide $\underset{\sim}{\tau}=g(l)$ (independently of $k$ ). Instead of (5.7), we now get:

$$
\operatorname{nor}\left(q^{k+l}(\alpha, m)\right) \geq \min \left(M^{k}, \operatorname{nor}\left(q^{k}(\alpha, m)\right)\right)-\min (l, m-k) \cdot \varphi(<m) \cdot m(\delta+2) \cdot r(m),
$$

and $r(m)=1 /\left(m^{2} \varphi(<m)\right)$. So

$$
\min (l, m-k) \cdot \varphi(<m) \cdot m \cdot(\delta+2) \cdot r(m) \leq m^{2} \cdot \varphi(<m) \cdot r(m) \cdot(\delta+2) \leq \delta+2 .
$$

So if we assume that

$$
\operatorname{nor}(p(\alpha, m))>\delta+2 \text { for all } m \in \omega \text { and } \alpha \in \operatorname{supp}(p, m),
$$

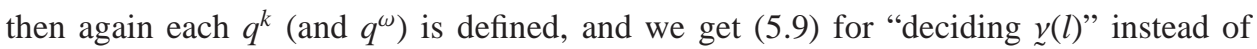
"essentially deciding $\tau$ ". But there is some $q^{\prime} \leq q^{\omega}$ deciding $\underset{\sim}{ }(l)$, a contradiction.

So far we know the following:

If $n$ is minimal trunk-length of $p$, if $p$ satisfies 5.10 , and if $M>2(\delta+2)$, then $S(p, n, M)$ exists and decides $v(n)$.

Rapid reading: Instead of the part on pure decision, we again proceed as follows: Fix $p \in P$ and $M>\delta+2$. We can assume that $p$ satisfies 5.10$)$, even for $2(\delta+2)$ instead of $\delta+2$ (just increase finitely many of the trunks). We set $k_{0}$ to be the minimal trunk-length of $p$, and $q^{k_{0}}=p$. We now construct $q^{k+1}$ and $q^{\omega}$ just as above, but this time using

$$
r^{i}=S\left(r^{i-1} \wedge s^{i}, k+1, M_{k}\right) .
$$

I.e. we try to decide $y(k+1)$. Each $r^{i}(\alpha, n)$ has sufficient norm, and so according to (5.11) $r^{i}$ (which has trunk-length $k+1$ ) decides $\underset{v}{q}(k+1)$. This implies that $q^{k+1}$ (and therefore $q^{\omega}$ as well) $\leq k$-decides $\underset{\sim}{\gamma}(k+1)$.

The rest of this section can safely be ignored: We describe how we end up with our particular definition of the product. We want to find a construction, similar to the countable support product, so that we can generalize the pure decision proof of Section 3.

- To get $\boldsymbol{\aleph}_{2}$-cc, the support of the product can be at most countable. For fusion, we have to allow at least countable support.

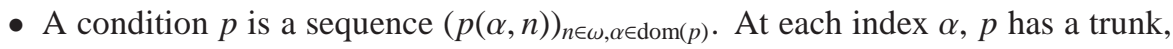
and above that $p(\alpha, n)$ is a creature in $\mathbf{K}_{\alpha}(n)$. 


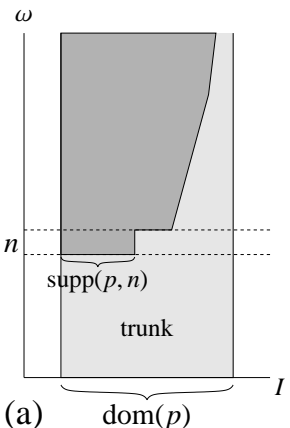

(a)

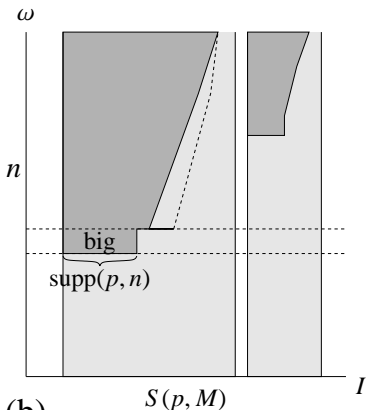

(b)

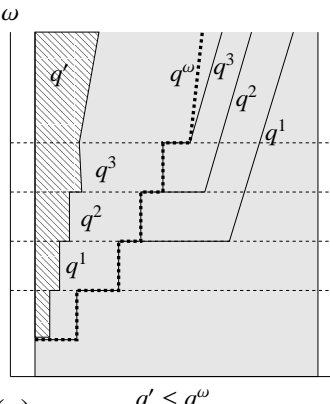

(c)

Figure 4. (a) A condition $p$ in $P$ : $\operatorname{dom}(p) \subseteq I$ is countable, at height $n$ there are less than $n$ many creatures. (b) The construction analog to $S(p, M)$. (c) We have to redefine $\leq$.

- To construct $S(p, M)$, we will set $n$ to be the minimal height of any stem of $p$. For each combination for values at height $n$ we get "dec" or "half". We want to use decisiveness to get homogeneous successors. For this we need that at height $n$, there are e.g. less than $n$ many creatures, and that $\mathbf{K}(n)$ is sufficiently decisive and big with respect to $n$. So we will generally assume that at each height $h$, there are less than $h$ many creatures, the rest is trunks, cf. Figure 4 (a).

- In the same construction step we also have to assume that each of the creatures at height $n$ has sufficient norm. So we will not just require that for each $\alpha \in I$ the norms of $p(\alpha, h)$ go to infinity, but that the minimum of all the norms at height $h$ go to infinity.

- When we set $q=S(p, M)$ and are in the case "half", instead of (3.12): "no $q^{\prime} \leq$ $q$ with trunk-length $n+1$ essentially decides $\underset{\sim}{\tau}$ ", we naturally get "no $q^{\prime} \leq q$ essentially decides $\underset{\sim}{\tau}$, if the trunk-length at $\alpha$ is the maximum of $n+1$ and the trunk-length of $p$ at $\alpha$."

- We now assume towards a contradiction that $q=S(p, M)$ is halving. We iterate the construction for all heights, get $q^{\omega}$, and find some $q^{\prime} \leq q^{\omega}$ essentially deciding $\underset{\sim}{\tau}$. However, this is not a contradiction: $q^{\prime}$ could just have a longer trunk at each $\alpha$, cf. Figure 4 (c).

- To fix this problem we redefine $q \leq p$ : We require that the trunk-lengths of $q$ are (on the common domain) almost always equal to those of $p$, cf. Figure 3 ,

- Once we redefine $q \leq p$ this way, and additionally require that at level $h$ there are less than $h$ many creatures, we could end up with a condition whose domain cannot be enlarged any more (since there already are maximally, i.e. $h-1$, many creatures at each level $h$ ). We fix this by adding e.g. the requirement that the number of creatures at level $h$ divided by $h$ converges to 0 .

\section{A DECISIVE CREATURE WITH BIGNESS AND HALVING}

In this section, we construct decisive creatures with halving.

We use $\mathbf{F}(n):=n$ for all $n$, i.e. the $n$-creatures live on the singleton $\{n\}$.

Lemma 6.1. Assume that $n$ and $B$ are natural numbers, and that $0<r<1$. Then there is a natural number $\Psi(n, B, r)$ so that we can set $\mathbf{H}(n)=\Psi(n, B, r)$ and find $r$-halving, $(B, r)$-big and $(n, r)$-decisive $n$-creatures $(\mathbf{K}(n), \mathbf{\Sigma})$ such that $\operatorname{nor}(\mathfrak{c})>n$ for some $\mathfrak{c} \in \mathbf{K}(n)$. 
Remarks. - Without the last requirement the lemma is trivial, just assume that nor $(\mathfrak{c})<$ 1 for all $\mathfrak{c} \in \mathbf{K}(n)$, and read the definitions of halving, big and decisive.

- If such $(\mathbf{K}(n), \boldsymbol{\Sigma})$ exists for some $\mathbf{H}(n)$, then it exists for every larger $\mathbf{H}(n)$ as well.

The rest of this section consists of the proof of the lemma. This proof is not needed in the rest of the paper.

We set rapidgrowth $(m)=2^{2^{m^{2}}}$ and $a:=2^{\frac{1}{r}}$. So $\log _{a}(2)=r$.

\section{The pre-norms.}

Lemma 6.2. There is a $J \in \omega$ and a function preprenor on the powerset of $J$ such that the following holds:

(1) preprenor is monotonous, i.e. $u_{1} \subseteq u_{2}$ implies preprenor $\left(u_{1}\right) \leq \operatorname{preprenor}\left(u_{2}\right)$.

(2) $\operatorname{preprenor}(\emptyset)=0$, and $\operatorname{preprenor}(J) \geq a^{n+1}$.

(3) If $\operatorname{preprenor}(u)=k+1$ then there is an $M \in \omega$ and a sequence $0=j_{0}<j_{1}<\cdots<$ $j_{M}$ such that $M \geq \max \left(B, \operatorname{rapidgrowth}\left(j_{1}+n\right)\right)$ and $\operatorname{preprenor}\left(u \cap\left[j_{i}, j_{i+1}-1\right]\right) \geq k$ for all $i \in M$.

Proof. For finite subsets $u$ of $\omega$ define preprenor $(u) \geq k$ by induction on $k$ : For all $u$ set preprenor $(u) \geq 0$, and $\operatorname{preprenor}(u) \geq 1$ iff $u$ is nonempty. For $k \geq 1$, we set preprenor $(u) \geq$ $k+1$ iff (3) as above holds. We show by induction on $k$ that for every $a \in \omega$ there is a $b \in \omega$ such that preprenor $([a, b-1])=k$ : Assume this is true for $k$. Given $a=j_{0}$, let $j_{1}$ be minimal such that $\operatorname{preprenor}\left(\left[j_{0}, j_{1}-1\right]\right)=k$. For every $i<\max \left(B, \operatorname{rapidgrowth}\left(j_{1}+n\right)\right)$, find the minimal $j_{i+1}$ such that preprenor $\left(\left[j_{i}, j_{i+1}-1\right]\right)=k$. Then preprenor $\left(\left[j_{0}, j_{M}-1\right]\right)=k+1$. So we can pick $J$ such that preprenor $([0, J-1])=a^{n+1}$.

We set $\Psi(n, B, r)=\mathbf{H}(n)=2^{J}$. For a subset $c$ of $\mathbf{H}(n)$, we set

$$
\operatorname{prenor}(c):=\max \left\{\operatorname{preprenor}(u): u \subseteq J, c \uparrow u=2^{u}\right\},
$$

where $c \uparrow u$ is $\{b \uparrow u: b \in c\}$. So $d \subseteq c$ implies prenor $(d) \leq \operatorname{prenor}(c)$.

Lemma 6.3. Assume that $M \in \omega, J$ a set, $u \subseteq J, c \subseteq 2^{J}, c \uparrow u=2^{u}, c=\bigcup_{i \in M} c_{i}$, and that $u_{i}(i \in M)$ are pairwise disjoint subsets of $u$. Then $2^{u_{i}}=c_{i} \uparrow u_{i}$ for some $i \in M$.

Proof. Otherwise, for all $i \in M$ there is an $a_{i} \in 2^{u_{i}} \backslash\left(c_{i} \uparrow u_{i}\right)$. Let $b \in 2^{u}$ contain the concatenation of these $a_{i}$. Then $b \in c \uparrow u$, so $b \in c_{i} \uparrow u$ for some $i \in M$, and $a_{i} \in c_{i} \uparrow u_{i}$, a contradiction.

The creatures. An $n$-creature $c$ is a pair $(c, k)$ such that $c \subseteq \mathbf{H}(n), k \in \omega$ and $k \leq$ $\operatorname{prenor}(c)-1$. nor $(\mathfrak{c})$ is determined from $(c, k)$ by

$$
\operatorname{nor}(c, k):=\log _{a}(\operatorname{prenor}(c)-k) .
$$

For $n$-creatures $\mathfrak{c} \cong(c, k)$ and $\mathfrak{D} \cong\left(d, k^{\prime}\right)$ we define

$$
\left(d, k^{\prime}\right) \in \Sigma(c, k) \text { if } d \subseteq c \text { and } k^{\prime} \geq k .
$$

We now show that these creatures satisfy our requirements:

Proof of Lemma 6.1. It is clear that norms can be bigger than $n$ :

$$
\operatorname{nor}(\mathbf{H}(n), 0)=\log _{a}(\operatorname{prenor}(\mathbf{H}(n)))=\log _{a}(\operatorname{preprenor}(J)) \geq \log _{a}\left(a^{n+1}\right)=n+1 .
$$


Halving. Assume nor $(\mathfrak{c})>1$, i.e. $\operatorname{prenor}(c)-k>a>2$. We define

$$
\text { half }(c, k):=(c, k+\lfloor(\operatorname{prenor}(c)-k) / 2\rfloor) \text {. }
$$

Note that $\log _{a}(\lceil(\operatorname{prenor}(c)-k) / 2\rceil) \geq \operatorname{nor}(c, k)-\log _{a}(2)=\operatorname{nor}(c, k)-r$. So

$$
\operatorname{nor}(\operatorname{half}(c, k))=\log _{a}(\operatorname{prenor}(c)-k-\lfloor(\operatorname{prenor}(c)-k) / 2\rfloor) \geq \operatorname{nor}(c, k)-r \text {. }
$$

If $\left(d, k^{\prime}\right) \in \boldsymbol{\Sigma}($ half $(c, k))$ and $\operatorname{nor}\left(d, k^{\prime}\right)>0$, then

$$
\operatorname{prenor}(d) \geq k^{\prime}+1 \geq k+\lfloor(\operatorname{prenor}(c)-k) / 2\rfloor+1,
$$

and we can un-halve $\left(d, k^{\prime}\right)$ to $(d, k) \in \mathbf{\Sigma}(c, k)$ :

$$
\operatorname{nor}(d, k)=\log _{a}(\operatorname{prenor}(d)-k) \geq \log _{a}(\lfloor(\operatorname{prenor}(c)-k) / 2\rfloor+1) \geq \operatorname{nor}(c, k)-r,
$$

and $\operatorname{val}(d, k)=\operatorname{val}\left(d, k^{\prime}\right)=d$.

Bigness. Let $(c, l)$ be an $n$-creature and nor $(c, l)=x+r \geq r$. Let $u \subseteq J$ witness prenor $(c)=$ $a^{x+r}+l=2 a^{x}+l$. So there is an increasing sequence $\left(j_{i}\right)_{i \in M+1}$ such that $c \nmid u=2^{u}$ and

$$
M \geq \max \left(B, \operatorname{rapidgrowth}\left(j_{1}+n\right)\right), \text { and }
$$

$$
\text { preprenor }\left(u \cap\left[j_{i}, j_{i+1}-1\right]\right) \geq 2 a^{x}+l-1 \geq a^{x}+l \text { for all } i \in M \text {. }
$$

(If $x>0$, the last inequality is strict.)

Take any $F: c \rightarrow M$. Then $c=\bigcup_{i \in M} F^{-1}\{i\}$. We set $u_{i}:=u \cap\left[j_{i}, j_{i+1}-1\right]$ for $i \in M$. According to Lemma6.3 there is an $i \in M$ such that $F^{-1}\{i\} \uparrow u_{i}=2^{u_{i}}$. We set $d:=F^{-1}\{i\} \subseteq$ $c$. Since preprenor $\left(u_{i}\right) \geq a^{x}+l$ and $d \uparrow u_{i}=2^{u_{i}}$, nor $(d, l) \geq \log _{a}\left(a^{x}\right)=x=\operatorname{nor}(c, l)-r$. This shows that $(c, l)$ is $(M, r)$-big, and in particular $(B, r)$-big.

Decisiveness. Pick $(c, l) \in \mathbf{K}(n)$ such that $\operatorname{nor}(c, l)=x+r \geq r$. As above there is a witness $u \subseteq J, M$ and $\left(j_{i}\right)_{i \in M+1}$. Set $u^{-}:=u \cap\left[j_{0}, j_{1}-1\right]$. Let $d^{-} \subseteq c$ contain for every $a \in 2^{u^{-}}$exactly one $b \in c$ such that $b \uparrow u^{-}=a$. Then $|d| \leq 2^{j_{1}}=: K$ and (as above) $\operatorname{nor}\left(d^{-}, l\right) \geq \operatorname{nor}(c, l)-r$. So $\left(d^{-}, l\right)$ is a $K$-small successor of $(c, l)$.

It remains to be shown that there is a $K$-big successor $\left(d^{+}, l\right)$.

Let $F: c \rightarrow 2^{j_{1}}<M$ map $b$ to $b \uparrow j_{1}$. So as above there is an $i<M$ such that $F^{-1}\{i\} \uparrow u_{i}=2^{u_{i}}$ for $u_{i}:=u \cap\left[j_{i}, j_{i+1}-1\right]$. Obviously $i \neq 0$. Set $d^{+}:=F^{-1}\{i\}$. Pick any $\left(d^{\prime}, l^{\prime}\right) \in \boldsymbol{\Sigma}\left(d^{+}, l\right)$ with norm bigger than 1 . Let prenor $\left(d^{\prime}\right)$ be witnessed by $u^{\prime}, M^{\prime},\left(j_{i}^{\prime}\right)_{i \leq M^{\prime}}$. Then $u^{\prime} \cap j_{1}=\emptyset$ (since every $b \in d^{\prime}$ has the same $b\left\lceil j_{1}\right.$ ). So $j_{1}^{\prime}>j_{1}$, and (by the same argument as above) $\left(d^{\prime}, l^{\prime}\right)$ is (rapidgrowth $\left.\left(j_{1}+n\right), r\right)$-big. This finishes the proof, since

$$
\text { rapidgrowth }\left(j_{1}+n\right)=2^{2^{\left(j_{1}+n\right)^{2}}} \geq 2^{2^{j_{1} \cdot n}}=2^{\left(2^{j_{1}}\right)^{n}}=2^{K^{n}} .
$$

\section{COUNTABLY MANY CARDINAL INVARIANTS}

Recall that $c_{f, g}^{\exists}$ and $c_{f, g}^{\forall}$ were defined in the introduction.

In the previous section, we defined $\Psi(n, M, r)$ for $r>0$ and $n, M \in \omega$. We can now specify the requirements we need for Theorem 1 ;

Assumption 7.1. $\left(f_{\epsilon}, g_{\epsilon}\right)_{\epsilon \in \omega}$ is a sequence of functions from $\omega$ to $\omega$. $f_{\max }$ is such that $f_{\epsilon}(m) \leq f_{\max }(m)$ for all $\epsilon \in \omega$. We set

$$
\varphi(=m):=f_{\max }(m)^{m}, \quad \varphi(<n):=\prod_{m<n} \varphi(=m) \quad r(n):=\frac{1}{n^{2} \varphi(<n)},
$$

and assume:

- If $\epsilon \neq \epsilon^{\prime}$, then there is an $n$ such that $f_{\epsilon}(m) \neq f_{\epsilon^{\prime}}(m)$ for all $m>n$.

- $f_{\epsilon}(m) \gg g_{\epsilon}(m)$ for all $\epsilon, m$; more precisely $f_{\epsilon}(m) \geq \Psi\left(m, g_{\epsilon}(m), r(m)\right)$. 
- If $f_{\epsilon}(m)>f_{\epsilon^{\prime}}(m)$, then $g_{\epsilon}(m) \gg f_{\epsilon^{\prime}}(m)$; more precisely $\varphi(<m) f_{\epsilon^{\prime}}(m)^{m}<g_{\epsilon}(m)$.

- $g_{\epsilon}(m)>\varphi(<m)$.

- $g_{\epsilon}(m+1) \geq f_{\max }(m)$ for all $\epsilon, m \in \omega$.

The assumption states more or less that the $f_{\epsilon}, g_{\epsilon}$ have sufficiently different growth rates, and that each level is much bigger than the previous levels. If is clear that we can construct such sequences (by induction).

Theorem 7.2. Assume CH. Choose for all $\epsilon \in \omega$ a cardinal $\kappa_{\epsilon}$ such that $\kappa_{\epsilon}=\kappa_{\epsilon}^{\kappa_{0}}$. Let $\left(f_{\epsilon}, g_{\epsilon}\right)_{\epsilon \in \omega}$ be as above. Then there is a proper, $\boldsymbol{\aleph}_{2}-c c, \omega^{\omega}$-bounding partial order $P$ which preserves cardinals and forces that $c_{f_{\epsilon}, g_{\epsilon}}^{\exists}=c_{f_{\epsilon}, g_{\epsilon}}^{\forall}=\kappa_{\epsilon}$ for all $\epsilon \in \omega$.

Let $I$ be the disjoint union of $I_{\epsilon}(\epsilon \in \omega)$ such that each $I_{\epsilon}$ has size $\kappa_{\epsilon}$ and is disjoint to $\omega$.

We will use $\epsilon, \epsilon^{\prime}, \epsilon_{1}, \ldots$ for the cardinal invariants (i.e. for elements of $\omega$ ), and $\alpha, \beta, \ldots$ for elements of $I$. $I$ will be the index set of the product.

So according to the definition of $\Psi$, we can choose for each $\epsilon, n \in \omega$ a creating pair $\left(\mathbf{K}_{\epsilon}(n), \boldsymbol{\Sigma}_{\epsilon}\right)$ satisfying the following:

- $\mathbf{F}_{\epsilon}(n)=n$,

- $\mathbf{H}_{\epsilon}(n)=f_{\epsilon}(n)$,

- $\mathbf{K}_{\epsilon}(n)$ is $\left(g_{\epsilon}(n), r(n)\right)$-big, $r(n)$-halving and $(n, r(n))$-decisive.

For every $\alpha \in I_{\epsilon}$ and $n \in \omega$, we set $\mathbf{K}_{\alpha}(n):=\mathbf{K}_{\epsilon}(n), f_{\alpha}:=f_{\epsilon}$ and $g_{\alpha}:=g_{\epsilon}$ and we set $\operatorname{trnklh}^{\min }(\alpha)$ to be the minimal $n$ such that $f_{\epsilon^{\prime}}(m) \neq f_{\epsilon}(m)$ for all $\epsilon^{\prime}<\epsilon$.

$P$ is the forcing notion defined in Section 5 , where we additionally require

- $\operatorname{trnklh}(p, \alpha) \geq \operatorname{trnklh}^{\min }(\alpha)$ for all conditions $p$ and $\alpha \in \operatorname{dom}(p)$.

As already noted, this does not change any of the results of Section 5 .

Note that $\varphi(<n)$ and $r(n)$ are as in Theorem 5.8, and that we assume CH. So we get:

Corollary 7.3. (1) $P$ is proper and $\boldsymbol{\aleph}_{2}-c c, P$ has continuous reading of names, and preserves all cardinals.

(2) (Separated support.) If $p \in P, \alpha, \beta \in \operatorname{supp}(p, n), \alpha \in I_{\epsilon}, \beta \in I_{\epsilon^{\prime}}$, and $\epsilon \neq \epsilon^{\prime}$, then $f_{\epsilon}(n) \neq f_{\epsilon^{\prime}}(n)$.

(3) (Rapid reading.) If $p \in P$ forces that $\eta$ is an $\left(f_{\epsilon}, g_{\epsilon}\right)$-slalom, or that $\eta(n)<f_{\epsilon}(n)$ for all $n$, then there is a $q \leq p$ which $\leq n$-decides $\eta(n)$ for all $n \in \omega$.

It also follows that $P_{\epsilon}:=P_{I_{\epsilon}}$ is a complete subforcing of $P$ and forces that the size of the continuum is $\kappa_{\epsilon}$.

Proof. (1): Theorem 5.8 and Lemma 5.4 (2): Assume that $\epsilon\left\langle\epsilon^{\prime} \cdot \operatorname{trnklh}(p, \beta)\right\rangle$ trnklh ${ }^{\min }(\beta)$, i.e. $f_{\epsilon}(n) \neq f_{\epsilon^{\prime}}(n)$. (3) follows from 5.9 Set $\delta=3, g(n)=f_{\max }(n-1)$ and $y(n)=\eta(n-1)$ for all $n$. Each $\mathbf{K}_{\epsilon}(n)$ is $\left(g_{\epsilon}(n), r(n)\right)$-big for some $\epsilon, g_{\epsilon}(n) \geq f_{\max }(n-1)=$ $g(n)$, and $p$ forces that there are at most $f_{\max }(n-1)^{f_{\max }(n-1)}<\operatorname{EXP}(g(n), n, 3)$ many possible

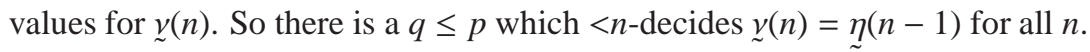

In the following two sections, we will show that $P$ forces $\kappa_{\epsilon} \leq c_{f_{\epsilon}, g_{\epsilon}}^{\exists}$ and $c_{f_{\epsilon}, g_{\epsilon}}^{\forall} \leq \kappa_{\epsilon}$. This proves Theorem 1 since $c_{f, g}^{\exists} \leq c_{f, g}^{\forall}$ for all $(f, g)$.

\section{8. $P_{\epsilon}$ ADDS A $\forall$-COVER}

Lemma 8.1. $P$ forces $c_{f_{\epsilon}, g_{\epsilon}}^{\forall} \leq \kappa_{\epsilon}$. 
One nice way to formulate the proof is the following: $P_{\epsilon}$ is a complete subforcing and forces $2^{\aleph_{0}}=\kappa_{\epsilon}$. And in the $P$-extension $V[G]$, the set of slaloms that are in the $P_{\epsilon}$-extension $V\left[G \cap P_{\epsilon}\right]$ form a $\left(\forall, f_{\epsilon}, g_{\epsilon}\right)$-cover.

However, to be able to generalize the proof to the uncountable case of Section 10, we will not use the complete subforcing. Instead we will use pure decision more explicitly.

Proof. Let $p_{0} \in P$ and $\underset{\sim}{r}$ be a $P$-name for a real such that $\underset{\sim}{r}(n)<f_{\epsilon}(n)$ for all $n$. We will show that

There is a $q \leq p_{0}$ and a way to determine an $\left(f_{\epsilon}, g_{\epsilon}\right)$-slalom $\underset{\sim}{S}(n)$ from $\operatorname{val}^{\Pi}(q, \leq n)$ restricted to $I_{\epsilon}$, such that $q$ forces $\underset{\sim}{r}(n) \in \underset{\sim}{S}(n)$ for all $n$.

More explicitly, we find a $q$ and a function eval which assigns to each $t \uparrow I_{\epsilon}$ for $t \in$ $\operatorname{val}^{\Pi}(q, \leq n)$ a set $S^{t}(n)$ such that $S^{t}(n) \subseteq f_{\epsilon}(n),\left|S^{t}(n)\right| \leq g_{\epsilon}(n)$ and such that $q$ forces the following: If $t$ is compatible with the generic filter, then $\underset{\sim}{r}(n) \in S^{t}(n)$.

Assume that we can do this for all names $\underset{\sim}{r}$. Note that there are only $\kappa_{\epsilon}$ many possible assignments as above: There are only $\kappa_{\epsilon}^{\kappa_{0}}=\kappa_{\epsilon}$ many possible sequences $q \uparrow I_{\epsilon}$, and $2^{\aleph_{0}}$ many ways to continuously read a real from $q \uparrow I_{\epsilon}$. Each assignment, together with the $P$-generic filter, determines a slalom $\underset{\sim}{S}$. Let $X$ be the set of all possible assignments. This corresponds to a $P$-name $Y$ of a family (of size $\kappa_{\epsilon}$ ) of $\left(f_{\epsilon}, g_{\epsilon}\right)$-slaloms, and according to 8.1 , the following holds in the $P$-extension: For every $\eta \in \prod_{n \in \omega} f_{\epsilon}(n)$ there is a slalom $\underset{\sim}{S}$ in $Y$ covering $\eta$. This implies $c_{f_{\epsilon}, g_{\epsilon}}^{\forall} \leq \kappa_{\epsilon}$.

So it remains to show (8.1). First pick a $p \leq p_{0}$ rapidly reading $\underset{\sim}{r}$ as in 7.3 (3), i.e. $p$ $\leq n$-decides $\underset{\sim}{r}(n)$ for all $n \in \omega$. We can assume that $\operatorname{nor}\left(p_{\alpha}(n)\right)>3$ for all $\alpha \in \operatorname{supp}(p, n)$. We set $\operatorname{dom}(q)=\operatorname{dom}(p)$ and $\operatorname{trnklh}(q, \alpha)=\operatorname{trnklh}(p, \alpha)$, and we will define $q(\alpha, m)$ (for all $\alpha \in \operatorname{supp}(p, m))$ as well as $\underset{\sim}{S}(m)$ by induction on $m$. We will find $q(\alpha, m) \in \boldsymbol{\Sigma}(p(\alpha, m))$ such that the norm decreases by at most 2 . Then $q$ automatically is a valid condition in $P$ and stronger than $p$.

Fix $m \in \omega$. Set $M:=\operatorname{supp}(p, m) \cap I_{\epsilon}$. (M stands for "medium".) According to "separated support' $7.3(2)$,

$$
\alpha \in \operatorname{supp}(p, m) \backslash I_{\epsilon} \text { implies } f_{\alpha}(m) \neq f_{\epsilon}(m) .
$$

So either $f_{\alpha}(m)<f_{\epsilon}(m)$, in this case we set $\alpha \in S$ (for "small"); or $f_{\alpha}(m)>f_{\epsilon}(m)$, then we set $\alpha \in L$ (for "large"). So $\operatorname{supp}(p, m)$ is partitioned into $S, M$ and $L$. We set $q(\alpha, m)=p(\alpha, m)$ for $\alpha \in S \cup M$.

$p \leq m$-decides $\underset{\sim}{r}(m)$, i.e. there is a function $F$ that calculates $\underset{\sim}{r}(m)<f_{\epsilon}(m)$ :

$$
F: \operatorname{val}^{\Pi}(p,<m) \times\left(\prod_{\alpha \in S \cup M \cup L} \operatorname{val}\left(p_{\alpha}(m)\right)\right) \rightarrow f_{\epsilon}(m) .
$$

Step 1: Assume $L$ is nonempty (otherwise continue with Step 2).

$$
\left|\prod_{\alpha \in S \cup M} \operatorname{val}\left(p_{\alpha}(m)\right)\right| \leq \mathbf{H}_{\epsilon}(m)^{m-1}=f_{\epsilon}(m)^{m-1} .
$$

So we can rewrite $F$ as

$$
F^{\prime}: \prod_{\alpha \in L} \operatorname{val}\left(p_{\alpha}(m)\right) \rightarrow f_{\epsilon}(m)^{\varphi(<m) f_{\epsilon}(m)^{m-1}}<f_{\epsilon}(m)^{f_{\epsilon}(m)^{m}} .
$$

If we set $B=\min \left(\left\{g_{\alpha}(m): m \in L\right\}\right)$, then $f_{\epsilon}(m)<B$, and $B^{B^{m}}<\operatorname{EXP}(B, m, 3)$. According to Corollary 4.4 there are $q(\alpha, m) \in \boldsymbol{\Sigma}\left(p(\alpha, m)\right.$ for $\alpha \in L$ such that $F^{\prime}$ restricted to $\prod_{\alpha \in L} \operatorname{val}(q(\alpha, m))$ is constant and $\operatorname{nor}(q(\alpha, m))>\operatorname{nor}(p(\alpha, m))-r(m) \cdot(m+3)$. This defines $q(\alpha, m)$ for $\alpha \in L$. So we now know $q(\alpha, m)$ for all $m$. 
Step 2: So (modulo $q$ ) we have eliminated the dependence of $\underset{\sim}{r}(m)$ on $L$, and are left with

$$
F: \operatorname{val}^{\Pi}(q,<m) \times\left(\prod_{\alpha \in S \cup M} \operatorname{val}(q(\alpha, m))\right) \rightarrow f_{\epsilon}(m) .
$$

We now define $\underset{\sim}{S}(m)$, more exactly the evaluation that maps $t \in \operatorname{val}^{\Pi}(q, \leq m) \uparrow I_{\epsilon}$ to $S^{t}(m)$. So fix such a $t \in \prod_{\alpha \in M} \operatorname{val}(q(\alpha, m))$.

$q \wedge t$ allows for at most $\varphi(<m) \cdot \prod_{\alpha \in S} \operatorname{val}(q(\alpha, m))$ many possible values for $\underset{\sim}{r}(m)$.

If $S$ is nonempty, let $\epsilon^{\prime}$ be such that $f_{\epsilon^{\prime}}(m)=\max \left\{f_{\alpha}(m): \alpha \in S\right\}$. Then $\prod_{\alpha \in S} \operatorname{val}\left(p_{\alpha}(m)\right) \leq$ $f_{\epsilon^{\prime}}(m)^{m}$. So we get $\varphi(<m) \cdot f_{\epsilon^{\prime}}(m)^{m}<g_{\epsilon}(m)$ many possible values for $\underset{\sim}{r}(m)$. (If $S$ is empty, we just get $\varphi(<m)$ many possibilities.) So we can set $S^{t}(m)$ to be this set of possible values.

\section{There IS NO SMALL $\exists$-COVER}

Lemma 9.1. (CH) $P$ forces $\kappa_{\epsilon} \leq c_{f_{\epsilon}, g_{\epsilon}}^{\exists}$.

Proof. Assume towards a contradiction that $p_{0}$ forces that $\mathcal{S}$ is an $\left(\exists, f_{\epsilon}, g_{\epsilon}\right)$-cover, $\aleph_{1} \leq$ $\lambda<\kappa_{\epsilon}$ and $\underset{\sim}{\mathcal{S}}=\left\{\underset{\sim}{\left.S_{i}: i \in \lambda\right\}}\right.$.

For every $i$, the set of $p^{\prime} \leq p_{0}$ which rapidly ${ }^{15}$ reads ${\underset{\sim}{i}}_{i}$ is predense under $p_{0}$. Because of $\boldsymbol{\aleph}_{2}$-cc, we can find a set $D_{i}$ of such $p^{\prime}$ which is predense under $p_{0}$ and has size $\boldsymbol{\aleph}_{1}$. So

$$
J=\bigcup_{i \in \lambda, p^{\prime} \in D_{i}} \operatorname{dom}\left(p^{\prime}\right)
$$

has size $\lambda$. Since $\left|I_{\epsilon}\right|=\kappa_{\epsilon}>\lambda$, there is a $\beta \in I_{\epsilon} \backslash J$. Fix this $\beta$.

Let $p_{1} \leq p_{0}$ decide the $i$ such that $\eta_{\beta}(n) \in \underset{\sim}{S_{i}}(n)$ for infinitely many $n$. We set $\underset{\sim}{S}:={\underset{\sim}{S}}_{i}$. We can assume $\beta \in \operatorname{dom}\left(p_{1}\right)$, so we have

$$
\beta \in \operatorname{dom}\left(p_{1}\right) \cap I_{\epsilon} \backslash J .
$$

Let $p \leq p_{1}$ be stronger than some $p^{\prime} \in D_{i}$, and let $\operatorname{nor}(p(\alpha, m))>10$ for all $\alpha \in \operatorname{supp}(p, m)$. So modulo $p$, we can determine the value of $\underset{\sim}{S}(n)$ from $t \uparrow J$ for $t \in \operatorname{val}^{\Pi}(p, \leq n) \underline{16}$

We will show towards a contradiction that we can strengthen $p$ to a $q$ such that for all $n \geq \operatorname{trnklh}(p, \beta)$ the following holds: the generic $\eta_{\beta}(n)$ (which is in $\operatorname{val}(q(\beta, n))$ and less than $f_{\epsilon}(n)$ ) avoids every possible element of $\underset{\sim}{S}(n)$, (which is determined by $q(\alpha, m)$ for $m \leq n$ and $\alpha \neq \beta$ ). In other words, we can make $\eta_{\beta}$ run away from $\underset{\sim}{S}$ at every height above the trunk. So $q$ forces that $\eta_{\beta}(n) \notin S(n)$ for all $n \geq \operatorname{trnklh}(p, \beta)$, a contradiction.

We set $\operatorname{dom}(q)=\operatorname{dom}(p), \operatorname{trnklh}(q, \alpha)=\operatorname{trnklh}(p, \alpha)$, and define $q(\alpha, m)$ (for all $\alpha \in$ $\operatorname{supp}(p, m))$ by induction on $m$. We will find a $q(\alpha, m) \in \boldsymbol{\Sigma}(p(\alpha, m))$ so that the norm decreases by at most 2. This guarantees that $q$ is a condition in $P$ and stronger than $p$.

Fix an $n \geq \operatorname{trnklh}(p, \beta)$. Set $A:=\operatorname{supp}(p, n)$. So $\beta \in A$, and without loss of generality $|A| \geq 2$. According to the definition of $P,|A|<n$.

Similarly to the previous section, we will partition $A$ into the large indices $L$, the small ones $S$ and $\{\beta\}$. However, we cannot assume that $A \cap I_{\epsilon}=\{\beta\}$, so the partition will not only be based on membership in $I_{\epsilon^{\prime}}$, but has to be "finer". $\underset{\sim}{S}(n)$ only depends on $S \cup L$ (and the very small set $\left.\operatorname{val}^{\Pi}(p,<n)\right)$. Again, we first use bigness to eliminate the dependence of $\underset{\sim}{S}(n)$ on the large part. And the small part is sufficiently small so that $\eta_{\beta}(n)$ (i.e. $q(n, \beta)$ ) avoids all the possible elements of $\underset{\sim}{S}(n)$. We now do this in more detail:

\footnotetext{
15 as in Corollary 7.3 3)

${ }^{16}$ More formally: Let $X$ be the set $\left\{t \uparrow J: t \in \operatorname{val}^{\Pi}(p, \leq n)\right\}$. For each $x \in X$ there is an $S_{n}^{x}$ such that $p$ forces: $(\forall \alpha \in J) x(\alpha)<\underset{\sim}{\eta_{\alpha}} \rightarrow \underset{\sim}{S}(n)=S_{n}^{x}$.
} 


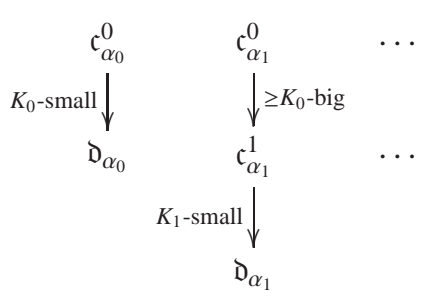

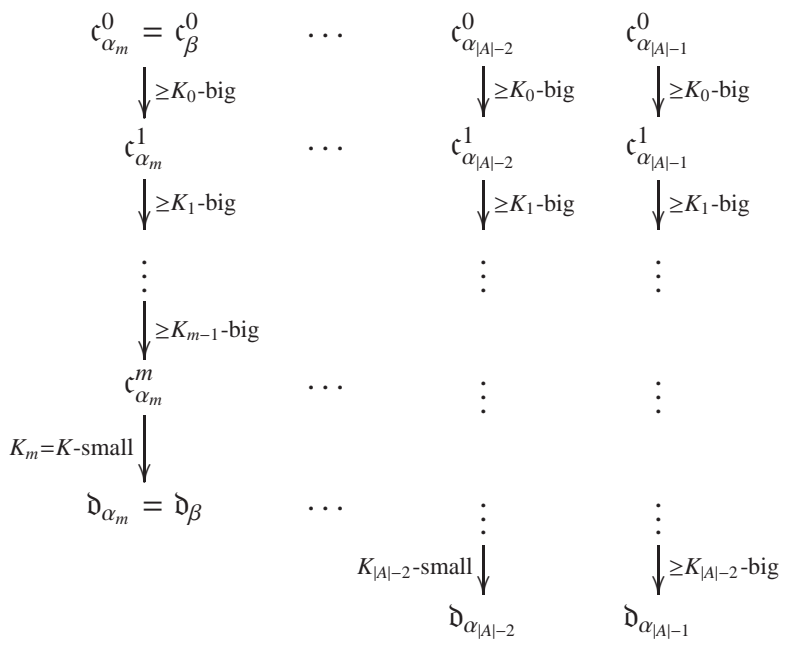

FiguRe 5.

Set $\mathrm{c}_{\alpha}^{0}:=p(\alpha, n)$ for $\alpha \in A$. Assume that for $l \geq 0$ we already have a list $\left(\alpha_{k}\right)_{k<l}$ of elements of $A$ and creatures $\left(c_{\alpha}^{l}\right)_{\alpha \in A \backslash\left\{\alpha_{0}, \ldots, \alpha_{l-1}\right\}}$. Each $c_{\alpha}^{l}$ is $\left(K_{\alpha}^{l}, n, r(n)\right)$-decisive for some $K_{\alpha}^{l}$. Set $K_{l}:=\min \left(\left\{K_{\alpha}^{l}: \alpha \in A \backslash\left\{\alpha_{0}, \ldots, \alpha_{l-1}\right\}\right\}\right)$, and choose $\alpha_{l}$ such that $K_{\alpha_{l}}^{l}=K_{l}$. Let $\mathrm{D}_{\alpha_{l}}$ be a $K_{l}$-small successor of $\mathrm{c}_{\alpha_{l}}^{l}$. For $\alpha \in A \backslash\left\{\alpha_{0}, \ldots, \alpha_{l}\right\}$, let $\mathrm{c}_{\alpha}^{l+1}$ be a $K_{l}$-big successor of $\mathrm{c}_{\alpha}^{l}$. Cf. Figure 5. Iterate this construction $|A|-1$ times. So there remains one $\alpha$ that has not been listed as an $\alpha_{l}$, set $\alpha_{|A|-1}=\alpha$ and $\mathrm{D}_{\alpha_{|A|-1}}=\mathfrak{c}_{\alpha}^{|A|-1}$.

Let $m$ be such that $\beta=\alpha_{m}$, and set

$$
K:=K_{m}, \quad S:=\left\{\alpha_{l}: l<m\right\}, \quad L:=\left\{\alpha_{l}: l>m\right\} .
$$

So $A$ is partitioned into the three parts $\{\beta\}, S$ and $L$. We get:

- $\mathfrak{D}_{\alpha} \in \boldsymbol{\Sigma}(p(\alpha, n)), \operatorname{nor}\left(\mathfrak{D}_{\alpha}\right) \geq \operatorname{nor}(p(\alpha, n))-(n-1) \cdot r(n)$.

- $\prod_{\alpha \in S}\left|\operatorname{val}\left(\mathrm{D}_{\alpha}\right)\right| \leq K_{m-1}^{n-2}<K$.

- $\mathrm{D}_{\beta}$ is hereditarily $K_{m-1}$-big $\sqrt{17}$ and $\left|\operatorname{val}\left(\mathrm{D}_{\beta}\right)\right| \leq K$.

- If $\alpha \in L$, then $\mathbb{D}_{\alpha}$ is hereditarily $K$-big 18

$J \cap \operatorname{supp}(p, n) \subseteq S \cup L$, so $S_{\sim}(n)$ is determined by $\operatorname{val}^{\Pi}(p,<n) \times \prod_{\alpha \in S \cup L} \operatorname{val}(p(\alpha, n))$. We set $q(\alpha, m)=\mathrm{D}_{\alpha}$ for all $\alpha \in S$. We also set $q(\beta, m)=\mathrm{D}_{m}$ for now. (But we may further decrease $q(\beta, m)$ in Step 2.) We are only interested in the elements of $\underset{\sim}{S}(n)$ that are possible values of $\eta_{\beta}(n)$, in other words we are interested in $\underset{\sim}{S}(n) \cap \operatorname{val}\left(\mathfrak{D}_{\beta}\right)$. This part has size at most $K$. So we get a function

$$
F: \operatorname{val}^{\Pi}(p,<n) \times\left(\prod_{\alpha \in S} \operatorname{val}\left(\mathrm{D}_{\alpha}\right)\right) \times\left(\prod_{\alpha \in L} \operatorname{val}\left(\mathfrak{D}_{\alpha}\right)\right) \rightarrow\left(\begin{array}{c}
K \\
g_{\epsilon}(n)
\end{array}\right) .
$$

Step 1: Assume $L$ is non-empty (otherwise continue with Step 2). Note that $\left(\begin{array}{c}K \\ g_{\epsilon}(n)\end{array}\right) \leq K^{g_{\epsilon}(n)}$ and $\varphi(<n)<g_{\epsilon}(n)<K$. So we can rewrite $F$ as

$$
F^{\prime}: \prod_{\alpha \in L} \operatorname{val}\left(\mathfrak{D}_{\alpha}\right) \rightarrow\left(K^{K}\right)^{K \times K}=K^{K^{3}} .
$$

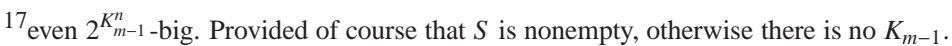

18 even $2^{K^{n}}$-big.
} 
Since $\mathfrak{D}_{\alpha}$ is decisive and (hereditarily) $K$-big for $\alpha \in L$ and $\operatorname{EXP}(K, n, 3)>K^{K^{3}}$, we can find $F^{\prime}$-homogeneous $q(\alpha, n) \in \mathbf{\Sigma}\left(\mathrm{D}_{\alpha}\right)$ for $\alpha \in L$ such that the norm decreases by at most $(n+1) \cdot r(n)$, cf. Corollary 4.4

Step 2: So modulo $q$ we have eliminated $L$ and can rewrite $F$ as

$$
F: \operatorname{val}^{\Pi}(p,<n) \times\left(\prod_{\alpha \in S} \operatorname{val}\left(\mathrm{D}_{\alpha}\right)\right) \rightarrow\left(\begin{array}{c}
K \\
g_{\epsilon}(n)
\end{array}\right) .
$$

Let $X$ be the image of $F$ (i.e. the set of possible values of $S_{\sim}(n) \cap \operatorname{val}\left(\mathrm{D}_{\beta}\right)$ ). $X$ has size at most $\varphi(<n) \cdot K_{m-1}^{n-2}<\operatorname{EXP}\left(K_{m-1}, n, 2\right)$. So according to 4.2 $(5)$, we can strengthen $\mathrm{D}_{\beta}$ to avoid $X$, decreasing the norm by at most $3 \cdot r(n)$.

\section{UNCOUNTABLY MANY INVARIANTS}

We construct natural numbers $\left(f_{n, l}\right)_{n \in \omega,-1 \leq l \leq n}$, and $\left(g_{n, l}\right)_{n \in \omega, 0 \leq l \leq n}$ so that $0=f_{0,-1}$ and (for $n, l \in \omega) f_{n+1,-1}=f_{n, n}$ and $f_{n, l-1}<g_{n, l}<f_{n, l}$. We set $f_{\max }(n)=f_{n, n}, \varphi(=n)=f_{\max }(n)^{n}$, $\varphi(<n)=\prod_{m<m} \varphi(=m)$ and $r(n)=1 /\left(n^{2} \varphi(<n)\right)$. So we get the following picture:

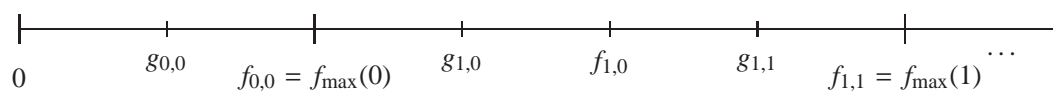

We require (for all $n, l \in \omega$ )

- $f_{n, l} \geq \Psi\left(n, g_{n, l}, r(n)\right)$ and

- $g_{n, l} \geq \varphi(<n) f_{n, l-1}^{n}$.

(Compare this with 7.1.) Again it is clear that we can construct such sequences by induction.

Let CHARS be the set of $v: \omega \rightarrow \omega$ such that $v(m) \leq m$ for all $m$. For $v \in$ CHARS, we can define $f_{v}: \omega \rightarrow \omega$ by $f_{v}(m)=f_{m, v(m)}$, and the same for $g_{v}$. So we assign to each $v \in$ CHARS cardinal characteristics $c_{f_{v}, g_{v}}^{\forall}$ and $c_{f_{v}, g_{v}}^{\exists}$.

Assume that $X \subset$ CHARS is countable such that

(10.1) for $v \neq v^{\prime}$ in $X$ there is an $n\left(v, v^{\prime}\right)$ such that $v(m) \neq v^{\prime}(m)$ for all $m>n\left(v, v^{\prime}\right)$.

Then $\left(f_{v}, g_{v}\right)_{v \in X}$ is a suitable sequence as in Assumption 7.1

Remark. We can of course find an uncountable set $X$ satisfying (10.1) as well. We could try to define a forcing $P_{I}$ just as in the countable case, to force an uncountable version of Theorem 11 However, we need "separated support" 7.3 (2) for 8.2). So we have to add appropriate requirements for conditions in $P$, in the style of trnklh ${ }^{\text {min }}$, this time depending on the pair $v, \nu^{\prime}$, to guarantee that the maximum of the trunk-lengths at $\alpha \in I_{v}$ and $\beta \in I_{\nu^{\prime}}$ is bigger than the $n\left(v, v^{\prime}\right)$. However, such requirements lead to the following problem: Assume that $Y \subseteq$ CHARS is countable and dense, and the domain of $p$ contains elements of $I_{v}$ for each $v \in Y$. Then we cannot enlarge the domain of $p$ to contain some $v^{\prime} \notin Y$ 19 So $p$ forces that the generic object does not contain anything in $I_{v^{\prime}}$. But then our proofs do not work any more, cf. e.g. (9.1). To fix this problem, we will modify the forcing $P$ in the

\footnotetext{
${ }^{19}$ In more detail: Let $f: Y \rightarrow \omega$ be such that for all $v \in Y$, there is an $\alpha \in \operatorname{dom}(p) \cap I_{v}$ such that $\operatorname{trnklh}(p, \alpha)+$ $1<f(v)$. Enumerate $Y$ as $\left\{v_{0}, v_{1}, \ldots\right\}$. Then construct $v^{\prime} \in$ CHARS $\backslash Y$ the following way: Pick any $v^{0} \in Y$ and pick a finite $v^{\prime 0}$ extending $v^{0} \uparrow f\left(v^{0}\right)$, such that $v^{\prime 0}(m) \neq v_{0}(m)$ for some $m$. Given $v^{\prime l}$, pick any $v^{l+1} \in Y$ extending $v^{\prime l}$, and pick $v^{\prime l+1}$ extending $v^{l+1} \uparrow f\left(v^{l+1}\right)$ such that $v^{\prime l+1}(m) \neq v_{l+1}(m)$ for some $m$. Set $v^{\prime}=\bigcup_{l \in \omega} v^{\prime l}$. Assume that there is a $q \leq p$ such that $\beta \in \operatorname{dom}(q) \cap I_{v^{\prime}}$ and $\operatorname{trnklh}(q, \beta)=m$. Only finitely many trunk-lengths in $\operatorname{dom}(p)$ were increased, so pick some $l$ such that $f\left(v^{l}\right)>m$ and such that not trunk in $I_{v^{l}}$ was increased. By the definition of $f, \alpha \in \operatorname{supp}(q, m)$ for some $\alpha \in I_{v^{l}}$. $v^{\prime}$ extends $v^{l} \uparrow f\left(v^{l}\right)$, so $v^{l}(m)=v^{\prime}(m)$ (and $\left.v^{l} \neq v^{\prime}\right)$, which contradicts separated support.
} 
following way: As before, we choose for each $\epsilon \in \omega_{1}$ a cardinal $\kappa_{\epsilon}$ and the index set $I_{\epsilon}$ of size $\kappa_{\epsilon}$. However, we do not fix a $\nu \in$ CHARS for $\epsilon$. Instead, each condition $p$ chooses $v(\epsilon)$ for each $\epsilon$ in its domain. This makes Theorem 2 slightly weaker than Theorem 1 since we do not know in the ground model which $v$ will be assigned to a $\kappa_{\epsilon}$.

We can now reformulate Theorem 2 .

Theorem 10.1. Assume $C H$, assume that $\kappa_{\epsilon}=\kappa_{\epsilon}^{\kappa_{0}}$ for $\epsilon \in \omega_{1}$, and that $\left(f_{v}, g_{v}\right)_{v \in C H A R S}$ are as above. Then there is a proper, $\boldsymbol{\aleph}_{2}-c c, \omega^{\omega}$-bounding partial order $R$ which forces: For each $\epsilon \in \omega_{1}$ there is a $v \in$ CHARS such that $c_{f_{v}, g_{v}}^{\forall}=c_{f_{v}, g_{v}}^{\exists}=\kappa_{\epsilon}$ for all $\epsilon \in \omega_{1}$.

(Here CHARS denotes the set in $V$, not the evaluation of the definition of CHARS in $V[G]$.

As in the proof of Section 7, we pick for each $v \in$ CHARS and $n \in \omega$ a creating pair $\left(\mathbf{K}_{v}(n), \boldsymbol{\Sigma}_{v}(n)\right)$, with $\mathbf{H}_{v}=f_{v}$ and $\mathbf{F}_{v}(n)=b$, which is $\left(g_{v}(n), r(n)\right)$-big, $r(n)$-halving and $(n, r(n))$-decisive.

We let $I$ be the disjoint union of $I_{\epsilon}\left(\epsilon \in \omega_{1}\right)$, each $I_{\epsilon}$ has size $\kappa_{\epsilon}$.

From here on, we assume $\mathrm{CH}$. We now define the forcing notion $R$ :

Definition 10.2. A condition $p$ in $R$ consists of a countable subset $\operatorname{dom}(p)$ of $I$, of objects $p(\alpha, n)$ for $\alpha \in \operatorname{dom}(p), n \in \omega$, and of functions $\operatorname{trnklh}(p): \operatorname{dom}(p) \rightarrow \omega$ and $\operatorname{char}(p):$ $\operatorname{dom}(p) \rightarrow$ CHARS satisfying the following $(\alpha, \beta \in \operatorname{dom}(p))$ :

- $\operatorname{char}(p, \alpha)=\operatorname{char}(p, \beta)$ iff $\alpha, \beta$ are in the same $I_{\epsilon}$.

- If $n<\operatorname{trnklh}(p, \alpha)$, then $p(\alpha, n) \in \mathbf{H}_{\mathrm{char}(p, \alpha)}(n)$. $\bigcup_{n<\operatorname{trnklh}(p)} p(\alpha, n)$ is called trunk of $p$ at $\alpha$.

- If $n \geq \operatorname{trnklh}(p, \alpha)$, then $p(\alpha, n) \in \mathbf{K}_{\mathrm{char}(p, \alpha)}(n)$ and $\operatorname{nor}(p(\alpha, n))>0$.

- $|\operatorname{supp}(p, n)|<n$ for all $n>0$.

- Moreover, $\lim _{n \rightarrow \infty}(|\operatorname{supp}(p, n)| / n)=0$.

- $\lim _{n \rightarrow \infty}(\min (\{\operatorname{nor}(p(\alpha, n)): \alpha \in \operatorname{supp}(p, n)\}))=\infty$.

- (Separated support.) If $\alpha, \beta \in \operatorname{supp}(p, n), \alpha \in I_{\epsilon}, \beta \in I_{\epsilon^{\prime}}$, and $\epsilon \neq \epsilon^{\prime}$, then $\operatorname{char}(p, \alpha)(n) \neq \operatorname{char}(p, \beta)(n)$.

$(\operatorname{supp}(p, n)$ is again the set of $\alpha \in I \operatorname{such}$ that $\operatorname{trnklh}(p, \alpha) \leq n$.

Another way to formulate the last point is: If $\alpha, \beta \in \operatorname{dom}(p), \alpha \in I_{\epsilon}, \beta \in I_{\epsilon^{\prime}}$, and $\epsilon \neq \epsilon^{\prime}$, then $\operatorname{char}(p, \alpha)$ and $\operatorname{char}(p, \beta)$ differ above some $n(\operatorname{char}(p, \alpha), \operatorname{char}(p, \beta))$ as in (10.1), and

$$
\max (\operatorname{trnklh}(p, \alpha), \operatorname{trnklh}(p, \beta))>n(\operatorname{char}(p, \alpha), \operatorname{char}(p, \beta)) .
$$

The order on $R$ is the natural modification of the one on $P$ :

Definition 10.3. For $p, q$ in $R, q \leq p$ if

- $\operatorname{dom}(q) \supseteq \operatorname{dom}(p)$,

- $\operatorname{char}(q, \alpha)=\operatorname{char}(p, \alpha)$ for all $\alpha \in \operatorname{dom}(p)$,

- if $\alpha \in \operatorname{dom}(p)$ and $n \in \omega$, then $q(\alpha, n) \in \Sigma_{\operatorname{char}(p, \alpha)}(p(\alpha, n))$,

- $\operatorname{trnklh}(q, \alpha)=\operatorname{trnklh}(p, \alpha)$ for all but finitely many $\alpha \in \operatorname{dom}(p)$.

$I_{\epsilon}$ is not a complete subforcing any more (conditions with disjoint domains are generally not compatible, since the union can violate separated support). But we still get:

Lemma 10.4. $R$ is $\aleph_{2}-c c$.

Proof. Assume towards a contradiction that $A$ is an antichain of size $\boldsymbol{\aleph}_{2}$. By a $\Delta$-system argument, we can assume that $\operatorname{dom}(p) \cap \operatorname{dom}(q)=u$ for all distinct $p, q$ in $A$. We fix an enumeration $\alpha_{0}^{p}, \alpha_{1}^{p}, \ldots$ of $\operatorname{dom}(p)$ for each $p \in A$. By a pigeon hole argument, we can 
assume that the following objects and statements are independent of $p \in A(n, i \in \omega, \beta \in u$, $\left.\epsilon \in \omega_{1}\right): " \alpha_{i}^{p}=\beta ", " \alpha \alpha_{i}^{p} \in I_{\epsilon}$ ", $\operatorname{trnklh}\left(p, \alpha_{i}^{p}\right), \operatorname{char}\left(p, \alpha_{i}^{p}\right)$, and $p\left(\alpha_{i}^{p}, n\right)$.

So given distinct elements $p, q$ of $A$, we again increase finitely many of the stems to guarantee that $\operatorname{supp}(p \cup q, n)$ has size less than $n$ for all $n$. Then the resulting $r$ is a condition in $R$ : To see e.g. separated support, assume that $\alpha, \beta \in \operatorname{supp}(r, n)$. We can assume that $\alpha=\alpha_{i}^{p}$ and $\beta=\alpha_{j}^{q}$ and that $\operatorname{char}\left(p, \alpha_{i}^{p}\right) \neq \operatorname{char}\left(q, \alpha_{j}^{q}\right)=\operatorname{char}\left(p, \alpha_{j}^{p}\right)$. Since $p$ satisfies separated support, $\operatorname{char}\left(p, \alpha_{i}^{p}\right)(n) \neq \operatorname{char}\left(p, \alpha_{j}^{p}\right)(n)$.

Lemma 10.5. $R$ adds a generic real $\eta_{\alpha}$ for all $\alpha \in I$. In other words, the set of conditions $q$ with $\alpha \in \operatorname{dom}(q)$ is dense.

Proof. Assume $\alpha \in I_{\epsilon}$. Fix $p \in R$. We find a $q \leq p$ with $\operatorname{dom}(q)=\operatorname{dom}(p) \cup\{\alpha\}$.

Case 1: $I_{\epsilon} \cap \operatorname{dom}(p) \neq \emptyset$. Then we pick $\beta \in I_{\epsilon} \cap \operatorname{dom}(p)$ and choose $\operatorname{trnklh}(q, \alpha)>$ $\operatorname{trnklh}(p, \beta)$ big enough to guarantee $|\operatorname{supp}(q, n)|<n$ for all $n$. Then we choose any $q(\alpha, n)$ with sufficient norm (e.g. $n)$.

Case 2: Otherwise we again fix $\operatorname{trnklh}(q, \alpha)$ big enough to guarantee $|\operatorname{supp}(q, n)|<n$ for all $n$, and we have to find some $\operatorname{char}(q, \alpha)$ satisfying separated support (for this $\operatorname{trnklh}(q, \alpha)$ ). Since $|\operatorname{supp}(p, n)|<n$ for all $n$, we can find a $v^{\prime} \in$ CHARS such that $v^{\prime}(n)$ is not in $\{v(n): v=\operatorname{char}(p, \beta), \beta \in \operatorname{supp}(p, n)\}$ for any $n$. Set $\operatorname{char}(q, \alpha)=v^{\prime}$. Then we again choose any $q(\alpha, n)$ with sufficiently increasing norms. $q$ satisfies separated support: Assume $\beta \in$ $I_{\epsilon^{\prime}} \cap \operatorname{supp}(p, n)$. Then $\operatorname{char}(p, \beta)(n) \neq v^{\prime}(n)=\operatorname{char}(q, \alpha)(n)$.

It turns out that the proofs of Theorems 5.8 and 5.9 still work without any change:

Lemma 10.6. $R$ is proper and $\omega^{\omega}$-bounding. If $\delta \in \omega, \underset{\sim}{\nu}(n)$ is a $P$-name and $p \in P$ forces that $\underline{v}(n)<\operatorname{EXP}\left(f_{\max }(n-1), n, n \cdot \delta\right)$ for all $n$, then there is a $q \leq p$ which $<n$-decides $y(n)$ for all $n$.

Proof. We define $\leq_{n}$ just as in the proof of Theorem 5.8. Fusion still works: If $q$ is the limit of $p_{n}$, and each $p_{n}$ satisfies separated support, then so does $q$. The proof of pure decision does not require any changes.

For rapid reading, note that each $\mathbf{K}_{v}(n)$ is $\left(f_{\max }(n-1), r(n)\right)$-big. Again, the same proof still works without changes.

We can define the $R$-name $\operatorname{char}(\epsilon)$ for $\epsilon \in \omega_{1}$ to be $\operatorname{char}(p, \alpha)$ for any $p$ in the generic filter and $\alpha \in \operatorname{dom}(p) \cap I_{\epsilon}$. Then we define the $R$-name $f_{\epsilon}$ to be $f_{\operatorname{char}(\epsilon)}$, and the same for $g_{\epsilon}$.

We again get all items of Corollary 7.3 and can show:

Lemma 10.7. $R$ forces $c_{f_{\epsilon}, g_{\epsilon}}^{\forall} \leq \kappa_{\epsilon}$ and $\kappa_{\epsilon} \leq c_{f_{\epsilon}, g_{\epsilon}}^{\exists}$.

Proof. The proofs of Lemmas 8.1 and 9.1 still work, if we assume that $p_{0}$ determines $\operatorname{char}(\epsilon)$.

\section{REFERENCES}

1. James E. Baumgartner, Iterated forcing, Surveys in set theory, London Math. Soc. Lecture Note Ser., vol. 87, Cambridge Univ. Press, Cambridge, 1983, pp. 1-59. MR MR823775 (87c:03099)

2. Martin Goldstern, Tools for your forcing construction, Set theory of the reals (Ramat Gan, 1991), Israel Math. Conf. Proc., vol. 6, Bar-Ilan Univ., Ramat Gan, 1993, available at http://info.tuwien.ac.at/goldstern/ pp. 305-360. MR MR1234283 (94h:03102)

3. Martin Goldstern and Saharon Shelah, Many simple cardinal invariants, Arch. Math. Logic 32 (1993), no. 3, 203-221. MR MR1201650 (94c:03064)

4. Jakob Kellner, Even more simple cardinal invariants, Arch. Math. Logic (to appear), cf. arXiv:0706.0319v2 http://arxiv.org/abs/0706.0319 
5. Andrzej Rosłanowski and Saharon Shelah, Norms on possibilities. I. Forcing with trees and creatures, Mem. Amer. Math. Soc. 141 (1999), no. 671, xii+167. MR MR1613600 (2000c:03036)

6. Saharon Shelah, Proper and improper forcing, second ed., Perspectives in Mathematical Logic, SpringerVerlag, Berlin, 1998. MR MR1623206 (98m:03002)

Kurt Gödel Research Center for Mathematical Logic, Universität Wien, Währinger Strasse 25, 1090 WIEN, AustRia

E-mail address: kellner@fsmat.at

URL: http://www.logic.univie.ac.at/ kellner

Einstein Institute of Mathematics, Edmond J. Safra Campus, Givat Ram, The Hebrew University of Jerusalem, Jerusalem, 91904, Israel, and Department of Mathematics, Rutgers University, New Brunswick, NJ 08854, USA

E-mail address: shelah@math.huji.ac.il

URL: http://shelah.logic.at/ 